\title{
X-Microscopy: Multicolor Super Resolution Image Reconstruction from Conventional Microscopy with Deep Learning
}

Lei $\mathrm{Xu}$

Shichao Kan

https://orcid.org/0000-0003-0097-6196

\section{Xiying Yu}

National Cancer Center/National Clinical Research Center for Cancer/Cancer Hospital, Chinese Academy of Medicaal Sciences and Peking Union Medical College

Yuxia Fu

National Cancer Center/National Clinical Research Center for Cancer/Cancer Hospital

\section{Yiqiang Peng}

HAMD (Ningbo) Intelligent Medical Technology Co., Ltd.

Yanhui Liang

HAMD (Ningbo) Intelligent Medical Technology Co., Ltd.

\section{Yigang Ceng}

Beijing Jiaotong University

Changjun Zhu

Tianjin Normal University

Wei Jiang ( $\nabla$ wjiang6138@cicams.ac.cn )

National Cancer Center/National Clinical Research Center for Cancer/Cancer Hospital https://orcid.org/0000-0002-2309-9571

\section{Article}

Keywords: X-Microscopy, Deep learning, SRM reconstruction, Cross-modality imaging

Posted Date: January 20th, 2022

DOI: https://doi.org/10.21203/rs.3.rs-1256986/v1

License: (c) (i) This work is licensed under a Creative Commons Attribution 4.0 International License.

Read Full License 


\section{X-Microscopy: Multicolor Super Resolution Image Reconstruction from Conventional Microscopy with Deep Learning}

Authors: Lei $\mathrm{Xu}^{1,2,6}$, Shichao Kan ${ }^{3,4,6}$, Xiying $\mathrm{Yu}^{1}$, Yuxia $\mathrm{Fu}^{1}$, Yiqiang Peng ${ }^{5}$, Yanhui Liang ${ }^{5}$, Yigang Cen ${ }^{4}$, Changjun Zhu ${ }^{2}$, Wei Jiang ${ }^{1,2,{ }^{*}}$

\section{Affiliations:}

${ }^{1}$ Department of Etiology and Carcinogenesis and State Key Laboratory of Molecular Oncology, National Cancer Center/National Clinical Research Center for Cancer/Cancer Hospital, Chinese Academy of Medical Sciences and Peking Union Medical College, Beijing, 100021, China

${ }^{2}$ Key Laboratory of Molecular and Cellular Systems Biology, College of Life Sciences, Tianjin Normal University, Tianjin 300387, China

${ }^{3}$ School of Computer Science and Engineering, Central South University, 410083, Changsha, Hunan, China ${ }^{4}$ Institute of Information Science, Beijing Jiaotong University, Beijing 100044, China

${ }^{5}$ HAMD (Ningbo) Intelligent Medical Technology Co., Ltd. Ningbo 315194, China

${ }^{6}$ These authors contribute equally to the work

*Corresponding author. Email: wjiang6138@cicams.ac.cn 
Deep learning shows an outstanding potential to transform low-resolved images into superresolved images. However, multicolor super resolution microscopy (SRM) imaging from wide- 


\section{Introduction}

Fluorescence microscopy (FM) plays a major role in monitoring cell physiology, especially the subcellular structures, molecular localizations and interactomes ${ }^{1}$. Using highly specified molecular probes together with developments of non-invasive and multi-color imaging capabilities, FM allows visualizations of complex biological systems ${ }^{2}$. Conventional FM imaging, however, has resolution barrier. Resolution was limited by the wavelength of light $\lambda$ and the numerical aperture (NA) of the objective lens ${ }^{3}$. Inventions of super-resolution microscopy (SRM) overcome the long-standing diffraction limit and continuously push the resolution barrier to the nanometer level, thus enabling visualization of previously invisible molecular details in biological systems that was previously only achievable with electron microscopy (EM) ${ }^{4,5}$.

Currently, SRM are improved on the bases of wide-field (WF), total internal reflection fluorescence (TIRF) and/or confocal microscopic setups. Although these setups in SRM imaging retain some advantages of conventional microscopy, each group of super-resolution techniques, such as photoactivated localization microscopy (PALM), stochastic optical reconstruction microscopy (STORM), stimulated emission depletion microscopy (STED) and structured illumination microscopy (SIM) ${ }^{6-9}$, has its own limitations. The sophisticated optical settings, expert handling, labeling probe and dye choices, considerations of sample characteristics for preparations, aberration correction, image mathematical post-processing and complex quantitative analyses are still required for SRM, making the fast, high-fidelity and high-throughput SRM imaging impossible.

Deep learning (DL) has had stunning successes in biomedical research and promises to revolutionize the microscopy field ${ }^{10-19}$. Consequently, several studies have established quick transformations from low resolution (LR) images to super resolution (SR) images by DL. Prior works using $\mathrm{DL}$ devoted to reconstructing SR views from LR images (i.e. confocal images) or under-sampled images or restore SIM images under low signal-to-noise ratio conditions ${ }^{20}$. These studies demonstrated that the $\mathrm{DL}$ technologies could help to overcome, at least in part, the disadvantages of SRM 21-26. However, fast and high-fidelity STORM-like SR images reconstructions from WFs with the conventional microscopy with DL have been hampered by accurate model establishments, large datasets of collections, exhaustive parameter search and computational cost. It remains uncertain whether reconstructing SR views using DL models outperform super resolution microscopy under some conditions.

Here, we report devising a deep network, X-Microscopy, a fusion of X-Net and UR-Net-8, which serves as transforming WFs to match the resolution acquired with STORM. X-Net leverages binary branches symmetrically and reciprocally to extract distinct fine details of two inputs. UR-Net-8, a core subnet of X-Microscopy, innovatively guides WFs to generate STORMlike under-sampled images in complex areas, providing excellent training data for X-Net. Once trained, X-Microscopy could extract features of WF that are hierarchically embedded into a reconstructed high-fidelity SRM image by channel attention and residual. Hence, X-Microscopy can easily, blindly and robustly reconstruct multi-color and size-flexible SRM images across different microscope modalities from WFs without trading off resolution. 


\section{Training and test datasets for X-Microscopy}

To determine whether X-Microscopy could restore SRM images (hereafter referred as SRMs) from WFs, commercial STORM system was applied to create datasets of immunofluorescence images, WFs from conventional microscopy and SRMs from STORM with PlanApo TIRF 100×/1.49 NA oil immersion objective. For each type of sample, not less than 30 sets of training and test pairs were obtained and divided proportionately. To provide demonstration of the network's generalization, We acquired datasets of WFs and paired SRMs of immunostained cytoskeletal MTs, F-actin microfilaments, MT plus-end binding protein, EB1 ${ }^{27}$, nuclear envelop structure protein, Lamin $\mathrm{B} 1{ }^{28}$, nucleosome histone protein $\mathrm{H} 2 \mathrm{~B}$ and histone $\mathrm{H} 3$ modification marker, H3K9me3 29-31, epithelial cytoskeletal intermediated filament (IF) component, Keratin 14 $(\mathrm{K}-14)^{32}$ and kinetochore antigen, CREST ${ }^{33}$ in different cell lines, HeLa, U2OS, RPE1, U87MG, U373MG and RNE-D3, with various fixed conditions (Methods). In this way, we trained the network to learn the pixel-to-pixel mapping from WFs to SRMs.

The training scheme on X-Microscopy was based on the popular generative adversarial network (GAN) method ${ }^{34-36}$ where the WFs were used as inputs to the net while the corresponding high-fidelity SRMs were defined as the ground-truth (G_T) targets, i.e., desired outputs. Each set of training data was averaged out to WF, STORM sparse (10000 consecutive frames from all the image sequence), STORM dense (95\% of all image sequence) and STORM perfect (all image sequence). All of WF-sparse-dense-perfect pairs were well matched and there was no need for pixel registration.

Collections of huge amounts of image data were extensive and time consuming. STORM images were typically acquired from no more than 30 FOVs (Fields of Views) for training. To prepare our datasets, the raw images were cropped into various size of perfect ROls (regions of interest) and then data augmentation algorithm was used to increase data diversities by random cropping. To prove the flexibility and generalization of our network, multiple test datasets were collected using different microscopic platforms and/or different image magnifications by different objective lens.

\section{The architecture of X-Microscopy and training strategy}

X-Microscopy, a synthesized pixel-to-pixel 35, 37 mapping network, was used for reconstruction of high-fidelity SRM images from WFs. X-Microscopy was a fusion of two subnets, X-Net and UR-Net-8, devised based on supervised DL ${ }^{38-40}$ (Figure 1A). Previously, Ouyang et al., demonstrated that a DL based ANNA-PALM network could reconstruct SRM images from WFs together with under-sampled images (sparse), achieving an MS-SSIM ${ }^{41} \approx 0.91$ when compared with standard STORM images ${ }^{22}$. If WFs and dense images were directly used as a training pair for high-fidelity SRM reconstruction by ANNA-PALM, the size of the training dataset had to be large enough to cover the distribution of images in the task domain. However, such huge amounts of training data were problematic because they were impossible to acquire. To circumvent the problem, we developed a new strategy where we first depicted a subnet, UR-Net-8, that 
innovatively guide WF to generate STORM-like under-sampled image (generated sparse) in complex areas with limited dataset (Figure 1B). In this way, UR-Net-8 was applied to generate sparse to replace the standard sparse for further image restoration task. Meanwhile, another subnet, X-Net, was designed to have two symmetrical and reciprocal branches that could extract distinct fine details of WFs and generated sparse for reconstructing high-fidelity SRM image by channel attention and residual ${ }^{42,43}$ (Figure 1C). Hence, X-Microscopy built by the fusion of URNet-8 and X-Net should utilize a minimal dataset for model training. Our goal was that, once trained, X-Microscopy was able to reconstruct SRMs rapidly and blindly from WFs alone without trading off resolution.

Inspired by U-Net ${ }^{44,45}$, the subnet of X-Microscopy, UR-Net-8, was devised for conducting sparse SRM generation. UR-Net-8 was stacked with 8 U-connected residual units, aiming to find predictive pixel relationships between sparse SRMs and WFs. UR-Net-8 was developed with 8 units by replacing the dehazing module with a general convolutional layer but kept input-size flexibility ${ }^{46}$. Each UR unit contained three convolutional layers, one de-convolutional layer, one channel concatenation layer and one residual layer. This design allowed UR-Net-8 to extract finer pixel-level features from WFs and reconstruct more abstract sparse structures (Figure S1A). Moreover, UR-Net-8 could learn the spatial interpolation rule that could best fit its task. To decrease and optimize learning parameters, two adjacent UR units in UR-Net-8 shared two convolutional layers, one channel concatenation layer and one residual layer.

For fast and high-fidelity SRM reconstruction from WF and generated sparse, the subnet of X-Microscopy, X-Net, was designed to have two inputs. Hence, X-Net could endow two computational paths, 1) an upper path extracted detailed features from WF with a deep stack of encoding units (encoder) and fused sparse features of lower path automatically and 2) a lower path extracted detailed pixel-level features from generated sparse with an encoder and fused WF features of the upper path automatically. These two paths were symmetrical and reciprocal that operated with a mutual-dependent fusion for acquiring, processing and fusing feature maps with fast generation of high-fidelity SRMs (Figure 1C).

By a carefully crafted design, 8 convolutional layers were added into X-Net encoder that allowed increasing the numbers of feature maps in the deeper layers, thus letting X-Net extract detailed and diversified pixel-level features from WFs and generated sparse. Meanwhile, each encoding unit performed one convolution using convolutional kernels with step size of 2 . This operation not only enabled the extractions of useful and comprehensive pixel-level information from WFs and/or generated sparse, but also realized the steadily reduction of the spatial resolution by 2 times, enforcing X-Net to learn more abstract structure-level features (Figure S1B).

To restore SRMs in terms of both sizes and resolution in X-Net decoder, convolutional kernels with step size of 2 were applied for performing de-convolution in each decoding unit (Figure S1B). We adopted channel attention and residual between each encoding unit and decoding unit. The attention unit was applied to refine important SRM features from the outputs of the connection that could determine what information should flow into the corresponding decoding units.(Figure S1C) The residual unit was utilized to suppress useless WFs and/or generated sparse features, which would filter out useful pixel-level information related to SRM from the 
encoding unit (Figures S1D).

To assist reciprocal learning ${ }^{47}$ and feature fusion between encoder and decoder, the output information from the encoder's symmetrical units between the channel attention and residual was combined in X-Net. Thus, the gradient information could flow reversely during the training phase and the information of each symmetric unit of two encoders could directly flow into the corresponding symmetric units of two decoders in the test stage, making reciprocal feature fusion and learning between encoders and decoders more effective for training and better SRM reconstruction(Figure S1B). Importantly, X-Net could pose extremely input-size flexibility characteristics, in which the feature maps in the convolutional layers and the deconvolutional layers were able to be calculated according to the sizes of the input images that were not applied previously in other networks (Table S1 and Table S2).

In addition, a discriminator was designed to assist training of X-Microscopy. During training, while UR-Net-8 and X-Net served as generators to generate computational SRMs from inputs, the discriminator, which consisted of four convolutional units, one spatial pyramid pooling unit and one linear classification unit, aimed to recognize whether the input SRM was real or generated (Figure S1E). The designed discriminator could then apply spatial pyramid pooling unit samples to fix length features from different sizes of input images and realize input-size flexibility.

A brief training strategy of X-Microscopy was illustrated as follows. The training processes of UR-Net-8 and X-Net were similar where an objective (also called loss) that contained two terms was applied to determine how accurate the output of UR-Net-8 or X-Net could match the desired output (G_T). The first term was defined as a combination of the absolute difference (L1 norm) and multi-scale version of the structural similarity index (MS-SSIM) ${ }^{41}$. Conditional GAN (cGAN) discriminator loss ${ }^{48}$ was adopted as the second term. WFs and/or generated sparse were served as inputs while the corresponding dense STORM images were defined as target outputs. The loss function of X-Net was akin to UR-Net-8, which could accelerate the convergence of the models and obtained desired reconstruction performance. UR-Net-8 and X-Net were implemented in TensorFlow to train the networks by end-to-end using stochastic gradient descent (SGD) with Adam optimizer. Since the optimizations of UR-Net-8 and X-Net from scratches typically took from hours to days on a single GTX Titan 1080 GPU, UR-Net-8 and X-Net were first trained from scratches with fixed size of images and then fine-tuned with different sizes of images, which could massively accelerate the optimization processes.

\section{Validating the feasibility of X-Microscopy.}

Previous study showed that SRMs could be reconstructed by a network, ANNA-PALM, using PALM-sparse images $(k=300 ; \Delta t=30 \mathrm{~ms})$ and/or WFs were in good agreement with PALM dense images. ANNA-PALM achieved a 100 -fold reduction of acquisition time compared to standard PALM ${ }^{22}$. To evaluate whether the trained X-Microscopy could fast output SRMs from sparse $(K=300 \sim 500)$ and WFs with high-fidelity, we compared X-Net and ANNA-PALM directly, which were trained with the same training and test datasets obtained by us. As shown in Figure 2A, MT images reconstructed from the trained X-Net revealed clear and super-resolved features of MTs that were not presented in WFs and sparse due to the low resolution and sparsity, 
coinciding to those of SRMs obtained by STORM. In contrast, although the trained ANNA-PALM also showed the effectiveness in SRM reconstructions, SRMs obtained by ANNA-PALM displayed some degrees of reconstruction reliability errors and artifacts in the complex areas. To further accurately define the similarities of SRMs reconstructed by the X-Net or by the ANNA-PALM with SRMs obtained by STORM, we calculated their values of structural similarity index (SSIM) ${ }^{49}$. SRMs reconstructed by the X-Net displayed SSIM that exceeded over 0.95, surpassing those of SRMs reconstructed by ANNA-PALM (Figure 2A).

We also merged paired MT SRMs reconstructed by X-Net and MT SRMs obtained by STORM to determine their coincidences (XK3 images as reconstructed SRMs and the dense images as G_T). Resolution quantification ${ }^{50}$ determined by decorrelation analyses indicated that MT SRMs reconstructed by the X-Net were similar with ground truth (perfect) while intensity profiles indicated that the $X-N e t$ provided minimal restoration errors (Figures 2A-2B). To assess the utility of the per-pixel predictions, we compared the true fluorescence pixel intensities for the network's predictions. Pearson correlation( $r$ ) values of ROls ${ }^{51}$ showed that they also exceeded over 0.95 values $(0.95$ and 0.96 , respectively), indicating that the network predicted pixel intensities were consistent with the SRM intensities obtained by STORM on per-pixel level (Figure 2C). Furthermore, the trained X-Net could accurately reconstruct high-fidelity SRMs from WFs of immunostained MTs from different cell lines and cell lines fixed by different methods (Figure S2AC). Moreover, we constructed SRMs from total MT datasets using the trained X-Net with inputsize flexibility and then systematically assessed their resolution and SSIM. Large-scale analyses of these MT SRMs constructed by the X-Net further demonstrated that they were akin to those of SRMs obtained by STORM (Figures 2D-2F). In addition, by utilization of random sampling sparse, the trained X-Net could resolve complex diffraction structures of MTs with at least 400× faster speed than STORM, which also outdistanced ANNA-PALM (Figure 2G). Taken together, these results demonstrated that images reconstructed by the trained X-Net from WFs and STORM sparse could be rapidly and accurately restored at a super-resolved level, similar to SRMs obtained by STORM.

\section{X-Microscopy can restore high-fidelity SRMs from WFs.}

As the goal of our aim was to restore high-fidelity SRMs from WFs alone with a small-scale dataset, we next determined whether SRMs could be reconstructed directly from diffractionlimited images obtained by a conventional microscope via the trained X-Net (Figure S3A). To this end, only WFs stained with MTs were used as inputs to the trained X-Net and blindly yielded super-resolved images as outputs. Although SRMs reconstructed by the trained X-Net exhibited clearly and curvilinearly MT structures that were not detailed in WFs, reconstruction errors could be easily detected in areas where the MT structures were complex and blurred (Figure S3B). Consistently, the quantitative analysis of SSIM, line profile and surface plot demonstrated that SRMs reconstructed from WFs were not as good as SRMs predicted from WFs plus STORM sparse (Figure S3C). Similar results were also obtained by ANNA-PALM from pervious study ${ }^{22}$. Hence, these results corroborated that applying STORM sparse $(K=300 \sim 500)$ as the guides was 
an essential factor for reconstructing structural-complex high-fidelity SRMs from WFs with a small-scale training dataset.

Therefore, we hypothesized if we could generate sparse from WFs, similar to G_T sparse, to provide extra information (resolution) for final SRM reconstruction. To test the idea, we applied UR-Net-8 to generate super-resolved sparse from MT WFs and used the UR-Net-8 generated sparse acting as guides for reconstructing high fidelity MT SRMs only from MT WFs by X-Net. We trained UR-Net-8 using WFs as inputs and paired STORM sparse as the G_T targets. The trained UR-Net-8 could blindly transform MT filaments in WFs into super-resolved sparse that matched well with those obtained by STORM in different acquisition times. The results also revealed that use of high-dense sparse $(k=10000)$ rather than use of low-dense sparse $(k=300 \sim 500)$ for model training could provide a better and higher fidelity sparse generation based on sparsity acquisitions (Figure S3D).

We used two quantitative metrics (Image Color Merge Channels, Foreground pixel coordinate distribution) to validate the high-fidelity quality of generated sparse by UR-Net-8. Paired images were merged, and then, the foreground pixel coordinate distribution analysis was applied to coordinate WF, the generated sparse, G_T sparse and perfect of ROls (regions of interest). The pixel coordinate distributions of the generated sparse were well-fitting with G_T sparse and precisely fell on width ranges of MTs in WFs and perfects (Figure S3E-3F). These results indicated that the generated sparse could simulate the role of real sparse in final SRM reconstruction, guiding WFs to learn the correct pixel distributions (resolution).

We applied X-Microscopy to reconstruct SRMs directly and blindly from WFs representing various complex subcellular structures in different cell lines with different fixed conditions. After training, the SRMs reconstructed by the trained X-Microscopy revealed extraordinary astonishing features beyond diffraction-limited scale. The SRMs of MTs or F-actin microfilaments reconstructed by the trained X-Microscopy displayed high-fidelity details that could detect not only in the areas with sparse distributed MTs or F-actin filaments but also the areas formed bifurcations, crisscrosses and less residuals (Figures $3 A$ and $3 C$, Figures S4A-S4E). Computational quantification analyses of the line profile and surface plot demonstrated that SRMs of MT or F-actin filaments reconstructed by the X-Microscopy were nearly the same, if not at all, as SRMs obtained by STORM (Figure 3B and 3C, Figures S4A and S4F and S4G). Statistical comparisons between the SRM reconstructions from WFs alone, the generated sparse only and WFs plus the generated sparse of MTs and F-actin filaments indicated that the generated sparse, indeed, assist in recovering the delicate high frequency details of super-resolved images (Figure S5F and 5G).

Besides reconstructions of SRMs from WFs stained with MT and F-actin tubular-like filaments, we next applied X-Microscopy to reconstruct SRMs of beehive-like structures of intermediate filaments (IFs) from WFs stained with K-14 in rat squamous epithelial cells, RNE-D3. Like MTs and F-actin filaments, IFs are also cytoskeletal constituents in cells but form more complex beehive-like structures. Reconstructions of SRMs of K-14 IFs from WFs clearly revealed K-14 IF networks that could elaborate fine K14 beehive-like filament nets in cells, which was unable to render in WFs. Quantification of the spatial relationship between SRMs and G_T 
images using SSIM indicated that SRMs of K-14 IFs reconstructed by trained X-Microscopy achieved over 0.9020 (Figure 3D). These results demonstrated that X-Microscopy could reconstruct SRMs from WFs with more complex cytoskeletal structures.

We applied X-Microscopy to reconstruct SRMs from WFs in other subcellular structures. SRMs of EB1 reconstructed by the trained X-Microscopy clearly revealed comet shapes of EB1 proteins with details not seen in WFs while SRMs of Lamin B1 predicted by the trained XMicroscopy displayed super-resolved details of the inner-nuclear envelop by eliminating many infocus backgrounds presented in WFs. The SSIM value of EB1 or Lamin B1 SRM obtained by the trained X-Microscopy achieved over 0.9660 and 0.9105 respectively (Figures $4 \mathrm{~A}$ and $4 \mathrm{C}$, Figure S5A-S5C). Pearson correlation( $r$ ) value (0.91) of XK3 (input=WF + generated sparse) indicated that the pixel intensities of XK3 (input=WF + generated sparse) were similar to G_T, showing the best reconstruction performance when compared with others (Figures 4B). Thus, the trained $X-$ Microscopy reconstructed SRMs of EB1 or Lamin B1 enabled us to determine structures and numbers of MT growing plus-ends or shapes and sizes of nuclei in a super-resolved level.

It was of interest that SRMs of kinetochores reconstructed by the trained X-Microscopy from WFs immunostained with CREST demonstrated that the X-Microscopy could compensate some of the STORM imaging limitations, such as phototoxicity and photobleaching, to precisely reconstruct SRMs for finer subcellular structures/macromolecules, i.e., paired sister-kinetochores. Paired sister-kinetochores in SRMs obtained by STORM often showed uneven acquisitions due to long-time STORM imaging and photobleaching although they could be separated well when compared with blurred paired sister-kinetochores in WFs by imaging diffraction-limit. However, SRMs reconstructed by the X-Microscopy revealed that paired sister-kinetochores represented not only as two clear splits but also even paired dot structures (Figure 4D, Figures S5D-S5E). The quality of SRM reconstruction from WF, generated sparse only and WF + generated sparse with whole test dataset was quantified by SSIM metric. When applied WF and generated sparse as input, the reconstruction reliability was enhanced and potential reconstruction artifacts was alleviated. (Figure S5H- S5J). Consistently, when SRMs and WFs of MTs were acquired under the condition that the imaging buffer was about quenching, SRMs reconstructed by the XMicroscopy displayed more continuations of MT filaments with fewer construction errors than SRMs obtained by STORM (Figure 4E).

Finally, X-Microscopy was challenged to reconstruct SRMs from WFs stained by antinucleosome histone $\mathrm{H} 2 \mathrm{~B}$ and $\mathrm{H} 3$ modification, $\mathrm{H} 3 \mathrm{~K} 9 \mathrm{me} 3$ antibodies in interphase cells, aiming if SRMs could be reconstructed from WFs with more complex subcellular structures, such as genome-wide higher-order chromatin structures. Consistent with published data ${ }^{29-31}$, perfect SRMs obtained by STORM revealed that $\mathrm{H} 2 \mathrm{~B}$ and $\mathrm{H} 3 \mathrm{~K} 9$ me3 staining displayed two major structural characteristics of higher-order chromatin, i.e. H2B staining that showed spatially segregated nucleosome nanoclusters and $\mathrm{H} 3 \mathrm{~K} 9 \mathrm{me} 3$ staining that revealed highly condensed aggregates. Reconstruction of SRMs from WFs were nearly identical to SRMs obtained by STORM. Qualitative visualization and quantitative analysis confirmed the structural similarity between SRMs and network output images. The SSIM values of H2B and H3K9me3 SRMs reconstructed by the trained X-Microscopy achieved over 0.9087 and 0.9014 , respectively (Figure 
3E and $3 F$ ). Thus, these results demonstrated that the trained X-Microscopy leveraged SRMs by STORM could blindly reconstruct SRMs only from WFs nearly to the G_T images.

\section{Multicolor SRM imaging with X-Microscopy.}

To determine whether X-Microscopy could predict high-fidelity multicolor SRMs ${ }^{52}$ from WFs, we first predicted SRMs of MTs and EB1 proteins by the trained X-Microscopy from WFs coimmunostained by Alexa Fluor 546 labeled anti- $\alpha$-tubulin antibodies and Atto 488 labeled antiEB1 antibodies. As shown in figure 5A, multicolor SRMs restored by the trained X-Microscopy displayed super-resolved structures where the MT signals gradually decayed from the peak position of EB1 toward their tips 53 (Figure 5B).

To further evaluate the utility and biological significance of X-Microscopy, we tested whether the trained X-Microscopy could fast and accurately reconstruct SRMs from WFs co-stained MTs and EB1 proteins under different experimental perturbations to assess MT dynamic instabilities ${ }^{54}$, 55. Wound-healing assay showed that EB1 comets on MTs in SRMs constructed by the trained XMicroscopy oriented clearly towards the direction of cell migration, which could be blocked by MT poising drugs, nocodazole and taxol. Cells treated with nocodazole or taxol displayed substantial alterations of the MT network in cells with drastic reductions of EB1 on MTs curved by nocodazole (depolymerization) or straightened by taxol (stabilization) (Figure S6).

We extended our study to restore multicolor SRMs from WFs co-immunostained with MTs and F-actin and WFs co-immunostained with MTs, Lamin B1 and kinetochore antigen, CREST. Multicolor SRMs predicted by the trained X-Microscopy clearly displayed transformation configurations, in which actin filaments were distributed around the periphery under the plasma membrane and formed bundles while MTs radiated from the MT organization center (MTOC) to form a dense network. Decorrelation analysis of reconstructed SRMs with WFs indicated that the resolution of SRMs could be improved by the X-Microscopy, nearly 3-4 times more than that of WFs (Figure 6A). Meanwhile, SRMs constructed by the trained X-Microscopy from WFs coimmunostained MTs, Lamin B1 and CREST, revealed that every feature of these subcellular structures could be transformed to a super-resolved level, where blurred, high-dense and/or indistinguishable MT network areas in WFs were resolved, Lamin B1 became more contoured and the distance between paired kinetochores could be clearly identified and determined (Figure $6 \mathrm{~B})$. Thus, these results demonstrated the excellent performance of the generic X-Microscopy in multicolor SRMs restoration.

\section{Cross-modality SRM imaging between different microscopic systems with X-Microscopy.}

For SRMs acquisition, such as SIM or STORM imaging, commercial super resolution microscopy systems were often designed with high power lenses (60x and 100x). To achieve high-throughput imaging and analyses, however, low power lenses were sometimes required for the practices. Thus, it was difficult to compromise between large FOVs imaging and high resolution due to low numerical aperture. Meanwhile, deep learning models could obtain high performance in inductive learning tasks, especially when the training and test datasets were 
sampled under similar experimental conditions. However, if test datasets were acquired from new categories, transfer learning was usually be applied for adapting the generic learned network to new datasets. Thus far, acquiring large FOVs of SRMs with low numerical aperture lenses for transfer learning was not possible.

To solve the problem, we tested whether X-Microscopy could achieve cross-modality SRM reconstruction by using different microscopic platforms and/or by using different magnification objective lenses without transfer learning. To this end, MT or Lamin B1 immunostained WFs were obtained by a Nikon conventional microscope with 20x, 40×, 60×, 100× objectives or by a Leica conventional microscope with a $100 \times / 1$.3NA objective. These WFs were used for SRM reconstruction by the trained X-Microscopy. As the X-Microscopy was trained from images obtained from a Nikon 100 /1.49 NA objective, a resizing algorithm was used to adjust images acquired from different objectives to almost the same conversion value of WF training datasets (Methods). Then these images were used for testing. As shown in figure 7 and figure S7, reconstruction qualities of SRMs by the X-Microscopy were gradually improved, i.e., the images obtained by lower magnification objectives or by a different type of microscopes, such as a Leica microscope, could be reconstructed to super-resolved levels by the X-Microscopy. These results indicated that X-Microscopy could be applied for cross-modality of SRM construction, thus providing an additional dimension for X-Microscopy application.

\section{Discussion}

In this study, we develop a deep neural network, X-Microscopy, powered by generative adversarial network (GAN) method ${ }^{34-36}$, which enables fast and high-fidelity reconstruction of SRMs on a single computer with/without GPU in a few seconds from WFs with input-size flexibility. Importantly, X-Microscopy requires no iteration and hyperparameter tuning during test phase, highlighting the scalability and cost-efficiency of X-Microscopy. Notably, X-Microscopy can be trained with limited availability of training data, which make accurate per pixel predictions of locations, precise intensities of subcellular complex structures and multicolor macromolecules, such as MTs, EB1, F-actin filaments, Lamin B1, K-14 IFs, histone H2B and H3K9me3 chromatin structures and kinetochores from WFs to SRMs. Thus, once trained, X-Microscopy can reconstruct multicolor and high-fidelity SRMs, which are otherwise required super-resolution microscopes with sophisticated optical settings, expert handling and limited labeling probe and dye choices, from WFs, which can be easily acquired from conventional microscopes with noninvasive and multi-color imaging capabilities, without trading off resolution. Furthermore, XMicroscopy can restore SRMs from WFs across different microscope modalities that add an additional dimension for X-Microscopy application.

Deep learning is becoming an increasingly important tool for SRM reconstruction and resolution enhancement in fluorescence microscopy. Thus far, many studies demonstrated that networks built on U-Net and generative adversarial network (GAN) architectures could leverage and transform low resolution (LR) images to super resolution (SR) images ${ }^{21-26,56}$. Trained an ANet designed with U-Net/GAN architectures, Ouyang et al., demonstrated that SRMs could be 
constructed by A-Net, also termed as ANNA-PALM, that had one input and one desired output with ingenious combination of $L 1$, structural similarity (SSIM) and conditional adversarial losses, fed with sparsely sampled PALM images and/or WFs to predict their densely sampled counterparts. Compared with PALM, ANNA-PALM improved spatial resolution and made considerable gains in acquisition time without dense fluorophores. However, when applied to SRM reconstruction from WFs alone, ANNA-PALM would produce errors. The frequency of errors could be reduced by using sparse as guides $(\geq 300){ }^{22}$. Weigert et al., introduced content-aware image restoration (CARE), a method that trained networks with low signal-to-noise ratio (input) and high signal-to-noise ratio (target) image pairs ${ }^{56}$. Using a similar network architecture and training loss, Wang et al., demonstrated that the resolution of diffraction-limited confocal images could be enhanced from $\sim 290 \mathrm{~nm}$ to $\sim 110 \mathrm{~nm}$ using only one biological structure with large-scale datasets ${ }^{25}$. Recently, Qiao et al., devised DFCAN and DFGAN, which leveraged the frequency content difference across distinct features to adaptively rescale their weightings when propagating them through the network and improve temporal resolution for live cell imaging ${ }^{23}$. However, the sizes of network's inputs and outputs were fixed for a trained model that would limit the application in variable-scale SRM reconstruction scenarios. In all, these state-of-the-art super resolution reconstruction methods that were utilized with one input and one desired output network showed limited successes in extracting independent fine-grain detailed features and realizing pixel mapping with various sizes of input images for SRM reconstruction with limited availability of dataset.

We took the challenges and devised X-Microscopy to close the gap of direct constructions of high-fidelity STORM-like images from WFs with a small-scale dataset. By fusion of highly efficient deep learning architectures based on pix2pix, i.e., a sparse generation network (UR-Net-8) and a home-built two branching network (X-Net), we demonstrated that X-Microscopy was able to leverage binary branches symmetrically and reciprocally to directly reconstruct WFs to superresolved STORM-like SRMs with limited dataset. We could achieve the goal because UR-Net-8 was devised for sparse generation to guide WFs to reduce artifacts in SRM predictions. Given the fact that UR-Net-8 was constructed by replacing the dehazing module with a general convolutional layer, which leverage shared layers to optimize the training parameters and simplify the training process ${ }^{46}$, it displayed an essential advantage for SRM reconstruction by keeping the input-size flexibility characteristic. We also incorporated residual into UR-Net-8 design to effectively suppress useless features for high-fidelity SRM reconstruction ${ }^{44}$. Hence, the lowresolution data conversion together with guidance afforded by UR-Net-8 alleviated the incompatibility between large-scale training datasets and STORM image acquisition times, facilitating super-resolved image reconstruction from WFs obtained from conventional microscopy.

As super-resolved generated sparse by UR-Net-8 in hands, X-Net was devised with two inputs, WFs and the generated sparse, for SRM reconstruction. Compared with previous studies where deep networks with one input shared convolution parameters and extracted features of different paired images based on similar convolutional kernels, which led to poor results with limited training dataset, X-Net comprised two encoders and two decoders, where two encoders could symmetrically and reciprocally extract independent fine-grained detailed features by 
learning distinctive convolutional kernels and hierarchically fused by cross-connections between encoding units and corresponding decoding units. Input independent attention was also adopted in each cross-connection module that could transform input-related salient features to precise representations of high-frequency information. Furthermore, unlike other published networks, the input sizes of X-Microscopy were flexible at both training and test stages by automatically calculating the sizes of feature maps based on various inputs. Thus, X-Microscopy has its great potential to advance the tasks of super-resolved image reconstruction with input-size flexibility by convention and/or super-resolution microscopy.

In supporting the conclusions, we first showed that super resolution comparable to standard STORM could be restored from sparse $(K=300 \sim 500)$ and/or WFs by X-Microscopy. The robustness of X-Microscopy was validated by 3 methods: a) predicting SRMs from WFs plus sparse in different cell lines and cell lines fixed by different methods; b) calculating average SSIM and resolution with large-scale test dataset; and c) comparing reconstruction qualities of STORM and X-Microscopy as function of frame number (or acquisition time). Thus, high-fidelity SRMs constructed by $X$-Microscopy with sparse $(k=100)$ were as fine as dense recorded by STORM $(K=40000)$. We then demonstrated that SRMs could be restored from WFs alone without relying on sparse images. When WFs was directly used as inputs to train or test, although artifacts in WFs were removed and resolution was enhanced by X-Microscopy, reconstruction errors could be easily detected in the areas where WFs were most ambiguous with subcellular structures having high densities and/or poor staining. Nevertheless, the problems could be solved by generated sparse using UR-Net-8. Generated sparse together with WFs could be used as inputs in X-Microscopy where generated sparse would guide WFs to distinguish fine details in complex area. Thus, SRMs inferred by X-Microscopy with large diversity of biological structures showed extraordinary astonishing features beyond diffraction-limited scale. Furthermore, SRMs reconstructed by X-Microscopy could overcome perturbations of WF acquisitions by phototoxicity, photobleaching and uneven imaging by buffer quenching. Moreover, the generalization and robustness of X-Microscopy were demonstrated by the multicolor SRM imaging from WFs. The resolution of X-Microcopy for multi-color SRM imaging could be 3-4 times better than that of WFs obtained by conventional microscopy. Finally, the ability of SRM reconstruction by X-Microscopy across different microscope modalities could push SRM into high-throughput and live-imaging applications, which might solve the bottleneck in acquiring large SRM datasets required for quantitative biology.

In summary, we illustrate X-Microscopy, a deep learning network, that can be applied for fast and accurate prediction of high-fidelity SRMs using WFs obtained from conventional microscopy. Hence, X-Microscopy has great potentials to enable widespread application for biological studies beyond what can be achieved by conventional microscopy in well-resourced institutions and laboratory settings.

\section{METHODS}

\section{KEY RESOURCES TABLE}


REAGENT or RESOURCE

antibodies

\begin{tabular}{|c|c|c|}
\hline Mouse anti- $\alpha$-tubulin & $\begin{array}{l}\text { Sigma- } \\
\text { Aldrich }\end{array}$ & T5168 \\
\hline Rat anti- $\alpha$-tubulin & $\begin{array}{l}\text { Sigma- } \\
\text { Aldrich }\end{array}$ & MAB1864 \\
\hline Mouse anti-EB1 & $\begin{array}{c}\text { BD } \\
\text { Pharmingen }\end{array}$ & 610535 \\
\hline Human anti-crest & $\begin{array}{c}\text { Cortex } \\
\text { Biochem }\end{array}$ & CS1058 \\
\hline Rabbit anti-Keratin 14 & Abcam & ab181595 \\
\hline Rabbit anti-H2B & Abcam & ab1790 \\
\hline Rabbit anti-H3K9me3 & Abcam & ab8898 \\
\hline Rabbit anti-LaminB1 & Abcam & ab1604 \\
\hline Alexa Fluor 647 phallodin & Invitrogen & A22287 \\
\hline Goat anti rat IgG Alexa fluor cy5 & Invitrogen & A21236 \\
\hline Goat anti mouse IgG Atto 488 & $\begin{array}{l}\text { Sigma- } \\
\text { Aldrich }\end{array}$ & 62197 \\
\hline Goat anti rabbit IgG Alexa fluor 546 & $\begin{array}{l}\text { Sigma- } \\
\text { Aldrich }\end{array}$ & A11010 \\
\hline Goat anti human IgG Alexa fluor cy5 & Invitrogen & A21445 \\
\hline Goat anti mouse IgG Alexa fluor 647 & Invitrogen & $\mathrm{A} 21236$ \\
\hline $\begin{array}{c}\text { Goat anti rat } \operatorname{lgG}(\mathrm{H}+\mathrm{L}) \text { Alexa Fluor } \\
568\end{array}$ & Invitrogen & A-11077 \\
\hline $\begin{array}{l}\text { Chemicals, Peptides, and } \\
\text { Recombinant Proteins }\end{array}$ & & \\
\hline DMEM/F-12 & Gibco & 11330032 \\
\hline RPMI 1640 & Gibco & 11875085 \\
\hline DMEM & Gibco & 11965092 \\
\hline Phosphate Buffer Saline & Gibco & 20012050 \\
\hline Trypsin-EDTA & Gibco & 25200072 \\
\hline Nocodazole & $\begin{array}{l}\text { Sigma- } \\
\text { Aldrich }\end{array}$ & SML1665 \\
\hline Taxol & $\begin{array}{l}\text { Sigma- } \\
\text { Aldrich }\end{array}$ & 444375 \\
\hline DMSO & $\begin{array}{l}\text { Sigma- } \\
\text { Aldrich }\end{array}$ & D8418 \\
\hline Fetal bovine serum & Gibco & 16140071 \\
\hline Cholera Toxin & $\begin{array}{c}\text { CELL } \\
\text { technologies }\end{array}$ & Cc104 \\
\hline insulin & $\begin{array}{c}\text { CELL } \\
\text { technologies }\end{array}$ & Cc101 \\
\hline hydrocortisone & $\begin{array}{c}\text { CELL } \\
\text { technologies }\end{array}$ & Cc103 \\
\hline EGF & PeproTech & $A F-100-15-500 \mu g$ \\
\hline Y27632 & Topscience & T1725 \\
\hline paraformaldehyde (PFA) & $\begin{array}{l}\text { Sigma- } \\
\text { Aldrich }\end{array}$ & 158127 \\
\hline Glutaraldehyde & $\begin{array}{l}\text { Sigma- } \\
\text { Aldrich }\end{array}$ & G6257 \\
\hline
\end{tabular}

SOURCE

IDENTIFIER

IIDNTIF 


\begin{tabular}{|c|c|c|}
\hline $\mathrm{NaBH} 4$ & $\begin{array}{l}\text { Sigma- } \\
\text { Aldrich }\end{array}$ & 71320 \\
\hline TritonX-100 & $\begin{array}{l}\text { Sigma- } \\
\text { Aldrich }\end{array}$ & T8787 \\
\hline Bovine Serum Albumin & $\begin{array}{l}\text { Sigma- } \\
\text { Aldrich }\end{array}$ & V900933 \\
\hline Goat serum & Solarbio & SL038 \\
\hline Sodium choride & $\begin{array}{l}\text { Sigma- } \\
\text { Aldrich }\end{array}$ & S9888 \\
\hline Tris-cl & $\begin{array}{l}\text { Sigma- } \\
\text { Aldrich }\end{array}$ & 10708976001 \\
\hline Glucose & $\begin{array}{l}\text { Sigma- } \\
\text { Aldrich }\end{array}$ & D9434 \\
\hline $\mathrm{HCl}$ & $\begin{array}{l}\text { Sigma- } \\
\text { Aldrich }\end{array}$ & 258148 \\
\hline ethanol & $\begin{array}{l}\text { Sigma- } \\
\text { Aldrich }\end{array}$ & 51976 \\
\hline methanol & $\begin{array}{l}\text { Sigma- } \\
\text { Aldrich }\end{array}$ & 34860 \\
\hline glucose oxidase & $\begin{array}{l}\text { Sigma- } \\
\text { Aldrich }\end{array}$ & G7141 \\
\hline catalase & $\begin{array}{l}\text { Sigma- } \\
\text { Aldrich }\end{array}$ & C9322 \\
\hline$\beta$-mercaptoethanol & $\begin{array}{l}\text { Sigma- } \\
\text { Aldrich }\end{array}$ & M3148 \\
\hline mercaptoethylamine (MEA) & $\begin{array}{l}\text { Sigma- } \\
\text { Aldrich }\end{array}$ & 30070 \\
\hline $\mathrm{NaOH}$ & $\begin{array}{l}\text { Sigma- } \\
\text { Aldrich }\end{array}$ & S588 \\
\hline $\mathrm{MgCl}_{2}$ & $\begin{array}{l}\text { Sigma- } \\
\text { Aldrich }\end{array}$ & M2393 \\
\hline \multicolumn{3}{|l|}{ Software and Algorithms } \\
\hline ImageJ & $\begin{array}{l}\text { Schneider et } \\
\text { al. } 2012\end{array}$ & https://imagej.nih.gov/ij/ \\
\hline Python2.7 & $\begin{array}{l}\text { Python } \\
\text { Software } \\
\text { Foundation }\end{array}$ & $\begin{array}{c}\text { https://www.python.org/download/releas } \\
\text { es/2.7/ }\end{array}$ \\
\hline Scipy package for Python & $\begin{array}{l}\text { Scipy } \\
\text { package for } \\
\text { Python }\end{array}$ & https://www.scipy.org \\
\hline $\begin{array}{l}\text { Tensorflow deep learning framework } \\
\text { for Python }\end{array}$ & Google Brain & https://tensorflow.google.cn/ \\
\hline Cuda & NVIDIA & $\begin{array}{l}\text { https://developer.nvidia.com/cuda- } \\
\text { downloads }\end{array}$ \\
\hline CuDNN & NVIDIA & https://developer.nvidia.com/cudnn \\
\hline GraphPad Prism 8 & GraphPad & $\begin{array}{l}\text { https://www.graphpad.com/scientific- } \\
\text { software/prism/ }\end{array}$ \\
\hline Adobe Illustrator 2020 & Adobe & https://www.adobe.com/ \\
\hline Adobe Photoshop 2019 & Adobe & https://www.adobe.com/ \\
\hline
\end{tabular}


Further information and requests for resources and reagents should be directed to and will be fulfilled by the Lead Contact, Wei Jiang (wjiang6138@cicams.ac.cn.).

\section{EXPERIMENTAL MODEL AND SUBJECT DETAILS}

\section{METHOD DETAILS}

\section{Cell culture}

Human cell lines were obtained from ATCC and cultured at $37^{\circ} \mathrm{C}$ with $5 \% \mathrm{CO}_{2}$ in humidified incubators. HeLa, U87MG and U2-OS cells were cultured in DMEM (Gibco) supplemented with 10\% FBS (BI) and 1\% penicillin-streptomycin antibiotics (Solarbio). RPE1 cells were maintained in DMEM/F12 medium (Gibco) supplemented with 10\% FBS and 1\% penicillin-streptomycin antibiotics. U373MG cells were grown in RPMI 1640 medium (Gibco) supplemented with 10\% FBS and $1 \%$ penicillin/streptomycin. Rat normal esophageal epithelial cell line, RNE-D3, was established and preserved by our laboratory. RNE-D3 was cultured in DMEM/F12 (3:1) medium supplemented with 10\% FBS, $8 \mathrm{ng} / \mathrm{mL}$ Cholera Toxin, $5 \mathrm{ng} / \mathrm{mL}$ insulin, $25 \mathrm{ng} / \mathrm{mL}$ hydrocortisone, $0.1 \mathrm{ng} / \mathrm{mL}$ EGF and $10 \mu \mathrm{M}$ Y27632 in a humidified $37^{\circ} \mathrm{C}$ incubator supplemented with $5 \% \mathrm{CO}_{2}$.

\section{Immunofluorescence}

For immunostaining, cells were cultured on glass-bottomed dishes (World Precision Instruments, Singapore) overnight and then washed three times with PBS. Cells were fixed either by adding $3 \%$ paraformaldehyde (PFA) and $0.1 \%$ glutaraldehyde solution in PBS at room temperature for 10 minutes or by adding acetone and methanol $(1: 1 \mathrm{v} / \mathrm{v})$ solution for $5 \mathrm{~min}$ at $20^{\circ} \mathrm{C}$. After washing with PBS three times, cells were treated with freshly prepared $0.1 \% \mathrm{NaBH}_{4}$ solution to bleach the autofluorescence for PFA fixation. Fixed samples were incubated in blocking buffer (10\% goat serum, 3\% BSA, 0.2\% Triton X-100 in PBS) overnight. Primary antibodies (mouse anti- $\alpha$-tubulin 1:50000, mouse anti EB1 1:5000, human anti CREST 1: 3000, rabbit anti Keratin 14 1:1000, rabbit anti H3K9me3 1:500, rabbit anti $\mathrm{H} 2 \mathrm{~B}$ 1:500 and/or rabbit anti Lamin B1 1:200) were added to the dish in blocking buffer. After 1-2 $\mathrm{h}$ incubation, cells were washed with washing buffer (0.2\%BSA, 0.02\% Triton X-100 in PBS) five times with at least 15 minutes of incubation between washes. Secondary antibodies (goat anti mouse/rat/human Alexa fluor 647 and/or goat anti-mouse Atto-488) were added at a dilution of 1:200 and incubated for 2 hours in blocking buffer on an orbital shaker. Before STORM immunofluorescence imaging, storing buffer was switched to the STORM imaging buffer $(50 \mathrm{mM}$ Tris- $\mathrm{Cl}, \mathrm{pH}=8.0,10 \mathrm{mM} \mathrm{NaCl}, 10 \%$ glucose) containing $10 \%$ (w/v) glucose (Sigma-Aldrich), $0.56 \mathrm{mg} / \mathrm{mL}$ glucose oxidase (SigmaAldrich), and $0.17 \mathrm{mg} / \mathrm{mL}$ catalase (Sigma-Aldrich) and $0.14 \mathrm{M} \beta$-mercaptoethanol (SigmaAldrich).

For wound healing assay and drug treatments, U2-OS cells were seeded in $35 \mathrm{~mm}$ glassbottomed dishes until reaching confluences. Confluent monolayers were scratched to create wounds using a $200 \mu \mathrm{L}$ sterile pipette tip. The monolayers were washed with fresh medium to 
remove debris and images of the wound areas were taken at 0 and $4 \mathrm{~h}$ using a $10 \times$ objective lens. Then, cells were treated with different concentrations of nocodazole (100 nM, Sigma-Aldrich) and taxol ( $1 \mu \mathrm{M}$, Sigma-Aldrich) for $30 \mathrm{~min}$. After drug treatments, cells were fixed and immunostained as described above.

\section{WFs and STORM images acquisition}

A commercial microscopic system from Nikon Instruments (N-STORM) was equipped with conventional microscopy and N-STORM at the same time, so WF images and SRM images could be recorded by the shared electron-multiplying charge coupled device (EMCCD) camera. All WFSRM pairs were well matched and there was no need for pixel registration, which simplified training process.

For training dataset, WFs were acquired by Nikon Eclipse Ti2 (Nikon) The exposure time was depended on dye concentration and incubation time. All images were recorded onto $512 \times 512$ or $256 \times 256$ pixel regions and 16 bit settings. To reduce image storage and speed up the training speed, all images were exported and saved as 8-bit as previous SRM reconstruction work 21, 22, 25. To evaluate the robustness of X-Microscopy, test images were acquired with Nikon Eclipse Ti2 and Leica DM5000 B.

All SRMs were acquired using Nikon N-STORM, an inverted microscope with PlanApo TIRF 100×/1.49 NA oil immersion objective (Nikon), four diode laser lines (405 nm, 488 nm, $561 \mathrm{~nm}$ and $647 \mathrm{~nm}$ ), W-view optics (Hamamatsu) and an EMCCD camera. The lasers were controlled directly by N-STORM computer software. All images were recorded onto $512 \times 512$ or $256 \times 256$ pixels region. The temperature of imaging environment was controlled at $23^{\circ} \mathrm{C}$.

For single-color STORM imaging (microtubule, actin, kinetochore, LaminB1 and EB1), $647 \mathrm{~nm}$ laser $(\sim 2 \mathrm{Kw} / \mathrm{cm} 2)$ was used to excite fluorescence from Alexa 647 molecules to switch them into the dark state and then $405 \mathrm{~nm}$ laser $(0-1 \mathrm{~W} / \mathrm{cm} 2)$ was used for image acquisition. In this way, at any given instant, only a small, optically resolvable fraction of fluorophores in the samples were in the emitting state. For STORM imaging of tubulin or actin, 50000-60000 frames were acquired at an exposure time of 10-20 ms. For STORM imaging of EB1 or kinetochore, 30000-50000 frames were acquired at an exposure time of 5-8 ms. For STORM imaging of LaminB1, 40000-50000 frames were recorded at an exposure time of $10 \mathrm{~ms}$. For STORM imaging of $\mathrm{H} 2 \mathrm{~B}, 40000-50000$ frames were recorded at an exposure time of $10-20 \mathrm{~ms}$. For STORM imaging of H3K9me3, 40000-50000 frames were recorded at an exposure time of $10 \mathrm{~ms}$. For STORM imaging of Keratin-14, 40000-50000 frames were recorded at an exposure time of 20 ms. All images were analyzed using NIS-Elements AR Analysis 5.01.00 (Nikon). Quantitative information for each localization, including the $x$ - and $y$-position, standard deviation $(\sigma)$, background noise and localization precision, was automatically determined by this procedure.

\section{Data preparation-The datasets of training and test for UR-Net-8.}

No more than 30 FOVs of different structures were acquired for training, respectively. For SRM reconstruction with deep learning, the size of the training dataset should be as large as 
possible to cover the distribution of images in the task domain for deep learning. However,

collecting huge amount of STORM SRM data can be time consuming and expensive. To achieve good performance with small-scale dataset, we therefore cropped the original image stacks into smaller ROls (regions of interest) manually to generate more training samples for all the experiments.

No more than 30 WF-sparse pairs of excellent ROls were applied for training. WFs were used as input and sparse images were defined as ground truth $\left(G_{-} T\right)$. To define sparse (G_T) images, random subsets of 10000 consecutive frames from all the image sequence were created. Each pair of images for training contained 30 sparse images, 1 widefield image and 1 perfect image. Detailed information about each training dataset was illustrated as follows. We trained our microtubule model on 30 ROls STORM images with corresponding widefield images. F-actin model was established with 14 ROls by transfer learning on microtubule model. The number of training samples for EB1 model was 15 ROls. For Lamin B1 model and kinetochore model, we trained with 17 ROls and 15 ROls, respectively. Keratin-14 model was constructed with 15 ROls. $\mathrm{H} 3 \mathrm{~K} 9$ me 3 model was established with 6 ROls and $\mathrm{H} 2 \mathrm{~B}$ model was trained with $8 \mathrm{ROIs}$. At the test stage, only widefield images were applied as inputs and the corresponding outputs were used for X-Net training.

\section{The datasets of training and test for X-Net.}

To validate the feasibility of X-Net for SRM reconstruction from sparse images and/or WFs, 30 pairs of immunostained microtubule images were used for X-Net training. To define sparse images, random subsets of 300-500 consecutive frames from all the image sequence were created. To define dense images, random consecutive subsets of $95 \%$ of all available frames were reconstructed. In addition, widefield images should be aligned with the corresponding dense images. Each pair of images for training contained 30 dense images, 30 sparse images, 1 widefield image and 1 perfect image. For test of X-Net, only widefield and sparse (300-500) images were used as inputs and the corresponding outputs were utilized against ground truth (perfect) images to calculate SSIM etc .

To demonstrate the robustness of X-Net, we trained and tested X-Net by random sampling sparse images, which were defined as $100,200,300,400,500,600,700,800,900,1000,1500$, $2000,3000,5000,10000,15000,20000,30000,40000$ consecutive frames.

To restore high-fidelity SRM images from WFs alone, we used the generated sparse predicted by UR-Net-8 and/or WFs as inputs for X-Net training. Detailed interpretation for each training dataset was similar with UR-Net-8.

\section{X-Microscopy-The architecture of UR-Net-8}

UR-Net-8, constructed based on skip-connection ${ }^{44}$ and residual ${ }^{42}$, was devised for conducting sparse SRM generation (Figure 1B). UR-Net-8 contained a generator network $\mathcal{G}_{s}$ and a discriminator network $\mathcal{D}_{s}$. For name interpretation of UR-Net-8, 'U' indicated U-connection, 'R' indicated residual, and ' 8 ' indicated 8 units of UR-Net used between the encoder and decoder 
(Figure S1A).

The generator network $\mathcal{G}_{s}$ comprised an encoder with 8 convolutional layers (encoding units) and a decoder with 8 de-convolutional layers (decoding units) (Figure 1B). Residual unit was utilized between each encoding unit and decoding unit. The architecture and operating principle of residual module were illustrated as follows. In the encoder, the number of feature channels was increased constantly by the first four convolutional layers, while was decreased by the last four de-convolutional layers in the decoder. From input layer to the output layer, the spatial resolution of feature maps in the encoder was halved layer by layer, while was doubled layer by layer in the decoder. In this way, the input of the UR-Net-8 passed through deeper convolutional layer of the encoder and more abstract feature maps were output. Then all output feature maps flowed directly into the adjacent encoding unit and the corresponding residual unit. Finally, the output of the residual unit flowed into the decoding unit. Convolutional kernels were applied to convolve feature maps in each convolutional layer. The convolutions of UR-Net-8 were adaptive zeropadded (i.e., the size of output feature maps was calculated based on the size of input image to decide whether zero-padding should be used). The kernel size of convolutional layers in the encoder and the de-convolutional layers in the decoder was $5 \times 5$ and the stride was 2 . Meanwhile, the convolutional layers in the residual units had convolutional kernels with size of $3 \times 3$ and stride of 1 (Figure S1A). Importantly, each convolution was followed by a rectified linear unit (ReLU) ${ }^{57}$ and batch normalization.

The $\mathcal{D}_{s}$ contained 4 convolutional layers (i.e., s1, s2, s3, and s4), 1 spatial pyramid layer (s5) and 1 linear classification layer (s6). s5 was a spatial pyramid pooling layer ${ }^{58}$, performing inputsize flexibility by sampling feature maps of different input sizes to vectors of the same length for the discriminator. The input of $\mathcal{D}_{s}$ was a concatenation of the input WFs of $\mathcal{G}_{s}$ and the generated sparse images along the channel dimension or a concatenation of two input images of $\mathcal{G}_{s}$ and the G_T image along the channel dimension. The output of $\mathcal{D}_{S}$ could be determined by training data and predefined ground truth values (Figure S1E).

\section{The architecture of X-Net}

X-Net was constructed to have a generator network $\mathcal{G}_{r}$ and a discriminator network $\mathcal{D}_{r}$ for performing adversarial training (Figure $1 \mathrm{C}$ ). The generator network $\mathcal{G}_{r}$ displayed two parallel networks with an X-shape to share information between encoders and decoders (Figure S1B). The two desired inputs of $\mathcal{G}_{r}$, which could be set as the generated sparse predicted from UR-Net8 and/or WFs acquired from conventional microscopy, were brought into two encoders and output feature maps. Then feature maps could concatenate along the channel dimension and propagated to two decoders, respectively. Finally, the outputs were concatenated along the channel dimension and generated reconstructed SRMs by convolution operation with one convolutional layer.

The detailed architecture of X-Net was illustrated as follows. For the generator network $\mathcal{G}_{r}$, the $\mathcal{G}_{r}$ was composed of two encoders and two decoders. Each encoder and each decoder were devised with 8 convolutional layers and 8 de-convolutional layers, respectively (Figure S1B). The kernel size of the first two layers in encoders and the last two layers in decoders was $5 \times 5$ and the stride was 2 . Other layers had convolutional kernels with size of $3 \times 3$ and stride of 2 . The rectified 
linear unit $(\operatorname{ReLU})$ activation function $(x \leftarrow \max (x, 0))$ was used to comply non-linear mapping in all layers except for e8 and d1 (Figure S3A). The batch-normalization function was applied to carry out fast convergence in all layers except e1 and d8. The procedures for extracting and processing feature maps of X-Net were similar with UR-Net-8. For the architecture of discriminator $\mathcal{D}_{r}$, it was akin to $\mathcal{D}_{s}$.(Figure S1E)

The attention and $U$-connection residual modules were key design of X-Net. As shown in Fig. S1B, they were established between each pair of encoding unit ei and decoding unit d(9-i) $(1 \leqslant i \leqslant$ 8 ) based on skip connection, respectively (ei and di were marked in figure S3A). They played an important role in concentrating on the major features and information extracted from WFs and sparse images to reconstruct the main and detailed maps of the SRMs. The detailed architecture of attention and residual modules was described as follows.

Each attention module had two inputs ( $A$ and $B$ in Figure. S1C). For the attention between layer $i$ of the decoder and layer $9-i$ of the encoder, $A$ is the output of $d(i-1), B$ is the input of ei. The attention module consisted of 5 convolutional layers and 1 de-convolutional layer. Four convolutional layers (a1, a3, a5 and a8) had kernels with size of $1 \times 1$ and stride of 1 and the kernel size of the convolutional layer (a2) was $2 \times 2$ and the stride was 2 (Table S3). The deconvolutional layer (a6) had kernels with size of 3 and stride of 2. Importantly, we calculated the element-wise addition (a4) for the convolution outputs of $A$ and $B$ (the outputs of a3 and a2) and calculated the element-wise multiplication (a7) for the output of de-convolution and the convolution output of $B$ (the outputs of a8 and a2) (Figure S1C). The residual module had four inputs ( $A, B, C$ and $D$ in Figure. S1D). For the residual between layer $i$ of the decoder and layer 9$i$ of the encoder. A was the output of $d(i-1)$, B and $C$ were the input of $e(9-i)$ in the upper and lower encoders, respectively. $\mathrm{D}$ was the output of the attention module that located between layer $i$ of the decoder and layer $9-i$ of the encoder. The residual unit included 1 de-convolutional layer $(\mathrm{r} 1)$ and 1 convolutional layer ( $r 6)$, where the kernel size of de-convolutional layer was $3 \times 3$ and the stride was 2 . The convolutional layer had kernels with size of $1 \times 1$ and stride of 1 (Table S4). One element-wise additional operation was used to calculate the residual between the output of the last convolutional layer (r6) and the input B.

\section{The training strategy of X-Net.}

Experiments were conducted on a computer with 64GB memory and one 11GB GTX Titan 1080 GPU. The algorithms were implemented on 64-bit ubuntu 16.04 operating system with Python 3.6.4 and 1.13.1 version of Tensorflow. X-Net was trained by end-to-end using stochastic gradient descent (SGD) with Adam ${ }^{59}$ and a batch size of 1 with 100,000 or more iterations (backpropagation steps). The momentum term of Adam was set as 0.5. The initial learning rate was set as $2 \times 10^{-4}$. During training, the parameters of the generator and discriminator were updated alternatively. We updated the parameters of the discriminator once after updating the parameters of the generator 4 times. In order to speed up training, the model was first trained from scratch with fixed-size images, then fine-tuned with different sizes of images. The network training from scratch typically took from hours to days on a single GTX Titan 1080 GPU. Once trained, the network took only few seconds to reconstruct SRMs with input-size flexibility (corresponding to an FOV). 
Three loss functions were applied to train the network, i.e., $L_{1}, L_{2}$, and $L_{3}$ in Figure. $1 \mathrm{C}$. Training loss functions was a combination of MS-SSIM loss ${ }^{60}, l_{1}$ norm loss and generative adversarial loss. The MS-SSIM loss was calculated by using 1 minus the MS-SSIM between the reconstructed SRMs and the G_T SRMs. The $l_{1}$ norm loss $\left(l_{1-j}, j \in\{1,2,3\}\right)$ was the mean of the absolute difference between the reconstructed SRMs and the G_T SRMs. The generative adversarial loss contained one generator loss and one discriminator loss. The generator loss was calculated based on the output value of the discriminator. The discriminator loss $l_{D-X}$ was the cross-entropy between the G_T value ( 0 or 1$)$ and the output value of the corresponding discriminator. The MS-SSIM assessed the similarity index of the overall structure between the reconstructed SRMs $\hat{O}$ and the G_T image 0 , which was calculated as follows: resized an image to different scales and calculated structure $s_{j}(\hat{O}, O)$, luminance $l_{j}(\hat{O}, O)$, and contrast $c_{j}(\hat{O}, O)$ at each scale, respectively. Here, $s_{j}(\hat{O}, O), l_{j}(\hat{O}, O)$, and $c_{j}(\hat{O}, O)$ were formulated as follows:

$$
\begin{aligned}
& s_{j}(\hat{O}, O)=\frac{\sigma_{\widehat{O} O}+C_{1}}{\sigma_{\widehat{O}} \sigma_{O}+C_{1}}, \\
& l_{j}(\hat{O}, O)=\frac{2 \mu_{\widehat{O}} \mu_{O}+C_{2}}{\mu_{\widehat{O}}^{2}+\mu_{O}^{2}+C_{2}}, \\
& c_{j}(\hat{O}, O)=\frac{2 \sigma_{\widehat{O}} \sigma_{O}+C_{3}}{\sigma_{\widehat{O}}^{2}+\sigma_{O}^{2}+C_{3}},
\end{aligned}
$$

where $C_{1}=\left(K_{1} T\right)^{2}, C_{2}=\left(K_{2} T\right)^{2}$ and $C_{3}=C_{2} / 2$ are non-zero constants of small value, which are used to prevent the denominator from being close to zero. In all experiments, $K_{1}=0.01$ and $K_{2}=$ 0.03 . $\mathrm{T}$ is the dynamic range and is set to 1.0. $\mu$ and $\sigma$ are the mean and standard deviation in a Gaussian sliding window $(11 \times 11)$. Then, the MS-SSIM is defined as:

$$
\operatorname{MS}-\operatorname{SSIM}(\hat{O}, O)=\left[l_{M}(\hat{O}, O)\right]^{\alpha_{M}} \cdot \prod_{j=1}^{M}\left[c_{j}(\hat{O}, O)\right]^{\beta_{j}}\left[s_{j}(\hat{O}, O)\right]^{\gamma_{j}},
$$

where $\mathrm{M}$ is the highest scale factor, $\alpha_{M}, \beta_{j}$ and $\gamma_{j}$ are used to adjust the relative weighting of the measurements at different scales. The values of them are set as the following values in the experiments:

$$
M=5, \alpha=\beta=\gamma=[0.0448,0.2856,0.3001,0.2363,0.1333]
$$

Finally, the MS-SSIM loss is defined as follows:

$$
l_{m s-s s i m}=1-\mathrm{MS}-\operatorname{SSIM}(\hat{O}, O) \text {. }
$$

The $l_{1}$ norm loss is defined as:

$$
l_{1}=\mathbb{E}(|\hat{O}-O|),
$$

where $\mathbb{E}$ is a mean function, which is equal to the average value of all input images.

Suppose that the output of the discriminator is $\hat{y}$, the ground truth value is 0 and 1 for the input of $\hat{O}$ and 0 , respectively. The discriminator loss $l_{D}$ is defined as:

$$
l_{d}=\mathbb{E}[\log (\hat{y}(O))+\log (1-\hat{y}(\hat{O}))] \text {. }
$$

Moreover, in all the experiments, the generative adversarial loss for the generator is 
defaulted as same as all other GAN-based losses, which is defined as $\mathbb{E}[\log (1-\hat{y}(\hat{O}))]$.

\section{The training strategy of UR-Net-8.}

UR-Net-8 was trained based on CGAN ${ }^{35}$. The training loss function was the combination of MS-SSIM loss, $l_{1}$ norm loss and generative adversarial loss. The calculation formulas for these losses were the same as these calculation formulas in X-Net training.

In the training process of UR-Net-8, the parameter setting and training strategy were consistent with the $\mathrm{X}$-Net except for the following settings. The initial learning rate was set as $1 \times 10^{-4}$. We updated the parameters of the generator for 10 times and the parameters of the discriminator for once, alternatively.

\section{Input-size Flexibility}

To realize input-size flexibility, the image input size and output size of each convolutional layer and each de-convolutional layer were calculated based on the size of input image. Suppose that the size of input image was $\mathrm{h} \times \mathrm{w}$ and the auto-computed output size of feature map was $H_{i} \times W_{i}$ at layer ei or di, where i was the index number of convolutional layers or de-convolutional layers. The size calculating functions were defined as follows:

$$
H_{i}=\left\lfloor\frac{h-1}{2^{i}}+1\right\rfloor, W_{i}=\left\lfloor\frac{w-1}{2^{i}}+1\right\rfloor,
$$

where symbol [.] means round down (e.g., [1.3]=1, [1.9]=1).

The hyper-parameters of our networks were shown in Tables S1-S7. Table S1 and Table S2 showed the hyper-parameters of encoders and decoders in X-Net. Table S2, Table S3, Table S4 and Table S5 showed the hyper-parameters of attention module, residual module and discriminator in X-Net. Table S6 and Table S7 showed the hyper-parameters of encoder and decoder in UR-Net-8. Table S8 showed the hyper-parameters of residual module in UR-Net-8. In each table, input size and output size of each layer were auto-computed based on the size of input images.

\section{Quantitative Performances for X-Microscopy}

At the test stage, four metrics were used to evaluate the performance of X-Microscopy, including image resolution, Pearson's correlation coefficients (R value), 3D-surface plot and SSIM. The resolution of each cropped image was estimated using the Image Decorrelation Analysis plugin of ImageJ with the default parameter settings. We used the whole-cell image instead of the cropped patches to estimate the resolution. As for SSIM, we used the standard STORM images as the ground truth to calculate.

The SSIM between image $\hat{O}$ and image 0 were calculated as follows:

$$
\operatorname{SSIM}(\hat{O}, O)=\frac{\left(2 \mu_{\hat{O}} \mu_{O}+C_{1}\right)\left(2 \sigma_{\hat{O}} \sigma_{O}+C_{2}\right)}{\left(\mu_{\hat{O}}^{2}+\mu_{O}^{2}+C_{1}\right)\left(\sigma_{\hat{O}}^{2}+\sigma_{\hat{O}}^{2}+C_{2}\right)}
$$

where $\mu_{\hat{o}}$ and $\mu_{O}$ were the mean values of pixels of image $\hat{O}$ and image 0 , respectively. $\sigma_{\hat{o}}$ and $\sigma_{O}$ 
were the standard deviation of pixels of image $\hat{O}$ and image 0 , respectively. We applied a Gaussian sliding window $(11 \times 11)$ moves pixel-by-pixel across the whole image to calculate the SSIM score. At each position of Gaussian window, SSIM score was calculated individually. The final SSIM score was the mean of total SSIM scores at all positions. Note that the SSIM metric was only used to evaluate the structure similarity during test. At the training stage, we calculated the MS-SSIM loss by applying the SSIM metric to multi-scale images of reconstructed SRM and ground truth SRM. The details of MS-SSIM metric were descripted above.

As for quantifying the accuracy of per-pixel prediction, we adopted global intensity normalization and frequency counts. We globally normalize pixel intensities via dividing by 255.0 (the pixel range of our images was 8 bit). All pixels were unified to fall within [0.0, 1.0]. Then, we count the pixel numbers per 1000 pixels and recorded as frequency counts in the bins with width of 0.1 . For Pearson $\rho$ value, Suppose that we have two images $X$ and $Y$, with each had $\mathrm{n}$ pixels. We calculated the Pearson $\rho$ value based on the following formula:

$$
\rho(X, Y)=\frac{\sum_{i=1}^{n}\left(X_{i}-\bar{X}\right)\left(Y_{i}-\bar{Y}\right)}{\sqrt{\sum_{i=1}^{n}\left(X_{i}-\bar{X}\right)^{2} \sum_{i=1}^{n}\left(Y_{i}-\bar{Y}\right)^{2}}},
$$

where i was the index of pixels, $\bar{X}$ and $\bar{Y}$ were the pixel means of $X$ and $Y$, respectively.

The intensity of line profile and color boxes were measured using the Plot Profile plugin of ImageJ. Statistical analyses were performed using GraphPad Prism 8.0. 3D surface plot of all images was computed by interactive 3D surface plot in Fiji/ ImageJ.

\section{Data Augmentation}

It should be noted that data augmentation was conducted after data preparation and image size below $100 \times 100$ would be trained directly without data augmentation. If image size was larger than $100 \times 100$, data augmentation would be used to process these images for training. Our detailed explanation of data augmentation program was illustrated as follows:

During the training, we first randomly generated 4 integer numbers between 0 and 49 , denoted as $w_{0}, h_{0}, w_{1}$, and $h_{1}$. in each iteration. Then, for images with sizes larger than $100 \times 100$, we cropped a patch from the input images using the following formula:

$$
\mathbf{I} \leftarrow \mathbf{I}\left[h_{0}: h-h_{1}, w_{0}: \mathrm{w}-w_{1}\right]
$$

where $\mathrm{h}$ and $\mathrm{w}$ are the height and width of image tensor $\mathbf{I}$. The left arrow indicated assignment and values update. After random cropping, we normalized values of the cropped patches between 0 and 1 by performing $\mathbf{I} \leftarrow \mathbf{I} / 255.0$. Here, 255.0 means that the pixel range of our experimental images was 8 bits. It should be noted that we do not consider images with sizes smaller than $100 \times 100$ in our experiments. Suppose that the input image size was smaller than $100 \times 100$, e.g., $80 \times 80$. The random generated integer numbers were $w_{0}=48, h_{0}=48, w_{1}=48$, and $h_{1}=48$. Finally, $w-w_{1}=32<w_{0}, h-h_{1}=32<h_{0}$. Note: the starting and ending coordinates of random cropped images were determined by a computer program rather than human operation. If the starting 
coordinates randomly fell on the edge of the image without content, useless and low-quality

contents (background without any image content) in the cropped images would interfere with training performance. Hence, data augmentation was not applied for images with sizes below $100 \times 100$ due to the possibility of useless information from image background.

\section{Multi-Color Super-Resolution Image Alignments}

We found that the multi-color SRMs reconstructed from wide-field image by X-Microscopy could be misaligned. This is because the range of one pixel of wide-field image may map to a range of multiple pixels of perfect SRM image. Therefore, the multi-color super-resolution images obtained by image reconstruction algorithm sometimes be misaligned. In order to align these reconstructed images, we developed an image alignment and calibration algorithm. Suppose that two reconstructed image tensors $\boldsymbol{I}_{1}$ and $\boldsymbol{I}_{2}$ are misaligned, we iteratively computed the square error between the corresponding values of these two tensors by moving one tensor one pixel in an iteration. Finally, we moved one tensor to the position of the least square error to align another tensor. Although tensors $I_{1}$ and $I_{2}$ had different content, this method could effectively align multicolor SRMs. It should be noted that the misaligned scale between different multi-color SRMs was very small, thus we needed to iterate over the $10 \times 10$ pixel range to calculate the square error. The equation for calculating square error was illustrated as follows:

$$
\varepsilon_{m n}=\sum_{i=1}^{h-m} \sum_{j=1}^{w-n}\left[\boldsymbol{I}_{1}(i, j,:)-\boldsymbol{I}_{2}(i+m, j+n,:)\right]^{2},
$$

where $1 \leq \mathrm{m} \leq 10$ and $1 \leq \mathrm{n} \leq 10$ are the range of rectangles that traversed. The aligned coordinate $(x, y)$ is calculated as:

$$
(\mathrm{x}, \mathrm{y})=\arg \min _{(m, n)} \varepsilon_{m n},
$$

This means that we can move values of tensor $\boldsymbol{I}_{1}$ from position $(\mathrm{i}, \mathrm{j})$ to position $(\mathrm{i}+\mathrm{x}, \mathrm{j}+\mathrm{y})$ or move values of tensor $I_{2}$ from position $(i+x, j+y)$ to position (i, j) for image alignment.

\section{Image Resizing.}

To determine whether XM could achieve cross modality SRM reconstruction, we used bilinear interpolation algorithm to resize the test image into multiple scales to adjust images acquired from different objectives to almost the same conversion value of $100 \times$ training dataset $(0.05 \mu \mathrm{m} / 1 \mathrm{px})$ before testing. Supposed that there are four pixels $\left[q_{11}, q_{12} ; q_{21}, q_{22}\right]$, and the position of them are $\left(x_{1}, y_{1}\right),\left(x_{1}, y_{2}\right),\left(x_{2}, y_{1}\right)$, and $\left(x_{2}, y_{2}\right)$, respectively. Then, we could use the following equation to calculate the pixel value $\mathrm{q}$ in position $(\mathrm{x}, \mathrm{y})$, where $x_{1}<\mathrm{x}<x_{2}, y_{1}<\mathrm{y}<y_{2}$ :

$$
\begin{gathered}
\mathrm{q} \approx \frac{\left(x_{2}-x\right)\left(y_{2}-y\right)}{\left(x_{2}-x_{1}\right)\left(y_{2}-y_{1}\right)} q_{11}+\frac{\left(x-x_{1}\right)\left(y_{2}-y\right)}{\left(x_{2}-x_{1}\right)\left(y_{2}-y_{1}\right)} q_{21}, \\
+\frac{\left(x_{2}-x\right)\left(y-y_{1}\right)}{\left(x_{2}-x_{1}\right)\left(y_{2}-y_{1}\right)} q_{12}+\frac{\left(x-x_{1}\right)\left(y-y_{1}\right)}{\left(x_{2}-x_{1}\right)\left(y_{2}-y_{1}\right)} q_{22},
\end{gathered}
$$

\section{References}

1. Sigal, Y.M., Zhou, R. \& Zhuang, X. Visualizing and discovering cellular structures with super-resolution microscopy. Science 361, 880-887 (2018). 
Sanderson, M.J., Smith, I., Parker, I. \& Bootman, M.D. Fluorescence microscopy. Cold Spring Harb Protoc 2014 pdb top071795 (2014).

3. Schermelleh, L. et al. Super-resolution microscopy demystified. Nat Ce/l Bio/ 21, 72-84 (2019).

4. Schermelleh, L., Heintzmann, R. \& Leonhardt, H. A guide to super-resolution fluorescence microscopy. J Cel/ Bio/ 190, 165-175 (2010).

5. Stewart, M. \& Vigers, G. Electron microscopy of frozen-hydrated biological material. Nature 319, 631-636 (1986).

6. Betzig, E. et al. Imaging intracellular fluorescent proteins at nanometer resolution. Science 313, 1642-1645 (2006).

7. Gustafsson, M.G. Nonlinear structured-illumination microscopy: wide-field fluorescence imaging with theoretically unlimited resolution. Proc Natl Acad Sci U S A 102, 13081-13086 (2005).

8. Hell, S.W. \& Wichmann, J. Breaking the diffraction resolution limit by stimulated emission: stimulated-emissiondepletion fluorescence microscopy. Opt Lett 19, 780-782 (1994).

9. Rust, M.J., Bates, M. \& Zhuang, X. Sub-diffraction-limit imaging by stochastic optical reconstruction microscopy (STORM). Nat Methods 3, 793-795 (2006).

10. Angermueller, C., Pärnamaa, T., Parts, L. \& Stegle, O. Deep learning for computational biology. Mo/ Syst Bio/ 12, 878 (2016).

11. Arganda-Carreras, I. et al. Trainable Weka Segmentation: a machine learning tool for microscopy pixel classification. Bioinformatics 33, 2424-2426 (2017).

12. Eulenberg, P. et al. Reconstructing cell cycle and disease progression using deep learning. Nat Commun 8 , 463 (2017).

13. Hinton, G.E. \& Salakhutdinov, R.R. Reducing the dimensionality of data with neural networks. Science 313, 504507 (2006).

14. Kraus, O.Z. et al. Automated analysis of high-content microscopy data with deep learning. Mo/ Syst Bio/ 13, 924 (2017).

15. LeCun, Y., Bengio, Y. \& Hinton, G. Deep learning. Nature 521, 436-444 (2015).

16. Lu, A.X., Kraus, O.Z., Cooper, S. \& Moses, A.M. Learning unsupervised feature representations for single cell microscopy images with paired cell inpainting. PLoS Comput Bio/ 15, e1007348 (2019).

17. Pärnamaa, T. \& Parts, L. Accurate Classification of Protein Subcellular Localization from High-Throughput Microscopy Images Using Deep Learning. G3 (Bethesda) 7, 1385-1392 (2017).

18. Shkolyar, A., Gefen, A., Benayahu, D. \& Greenspan, H. Automatic detection of cell divisions (mitosis) in liveimaging microscopy images using Convolutional Neural Networks. Annu Int Conf IEEE Eng Med Biol Soc 2015, 743-746 (2015).

19. von Chamier, L., Laine, R.F. \& Henriques, R. Artificial intelligence for microscopy: what you should know. Biochem Soc Trans 47, 1029-1040 (2019).

20. Belthangady, C. \& Royer, L.A. Applications, promises, and pitfalls of deep learning for fluorescence image reconstruction. Nat Methods 16, 1215-1225 (2019).

21. Jin, L. et al. Deep learning enables structured illumination microscopy with low light levels and enhanced speed. Nat Commun 11, 1934 (2020).

22. Ouyang, W., Aristov, A., Lelek, M., Hao, X. \& Zimmer, C. Deep learning massively accelerates super-resolution localization microscopy. Nat Biotechno/36, 460-468 (2018).

23. Qiao, C. et al. Evaluation and development of deep neural networks for image super-resolution in optical microscopy. Nat Methods 18, 194-202 (2021).

24. Wagner, $\mathrm{N}$. et al. Deep learning-enhanced light-field imaging with continuous validation. Nat Methods 18, 557563 (2021).

25. Wang, H. et al. Deep learning enables cross-modality super-resolution in fluorescence microscopy. Nat Methods 16, 103-110 (2019).

26. Wu, Y. et al. Three-dimensional virtual refocusing of fluorescence microscopy images using deep learning. Nat 
Methods 16, 1323-1331 (2019).

27. Bieling, P. et al. Reconstitution of a microtubule plus-end tracking system in vitro. Nature 450, 1100-1105 (2007)

28. Padiath, Q.S. et al. Lamin B1 duplications cause autosomal dominant leukodystrophy. Nat Genet 38, 1114-1123 (2006).

29. $\mathrm{Xu}$, J. et al. Super-Resolution Imaging of Higher-Order Chromatin Structures at Different Epigenomic States in Single Mammalian Cells. Cell Rep 24, 873-882 (2018).

30. $\mathrm{Xu}, \mathrm{J}$. et al. Super-resolution imaging reveals the evolution of higher-order chromatin folding in early carcinogenesis. Nat Commun 11, 1899 (2020).

31. Zessin, P.J., Finan, K. \& Heilemann, M. Super-resolution fluorescence imaging of chromosomal DNA. J Struct Biol 177, 344-348 (2012).

32. Cheung, K.J. et al. Polyclonal breast cancer metastases arise from collective dissemination of keratin 14expressing tumor cell clusters. Proc Natl Acad Sci U S A 113, E854-863 (2016).

33. Miller, J.M., Wang, W., Balczon, R. \& Dentler, W.L. Ciliary microtubule capping structures contain a mammalian kinetochore antigen. J Cel/ Bio/110, 703-714 (1990).

34. Goodfellow, I.J. et al. in Advances in Neural Information Processing Systems 27: Annual Conference on Neural Information Processing Systems 2014 2672-2680 (2014).

35. Isola, P., Zhu, J.Y., T., Z. \& Efros, A.A. in 2017 IEEE/CVF Conference on Computer Vision and Pattern Recognition (CVPR) 5967-5976 (2017).

36. Zhu, J.Y., Park, T., Isola, P. \& Efros, A.A. in 2017 IEEE International Conference on Computer Vision 2242-2251 (2017).

37. Huang, X., Liu, M.Y., Belongie, S. \& Kautz, J. Multimodal Unsupervised Image-to-Image Translation. European Conference on Computer Vision (2018).

38. He, K., Zhang, X., Ren, S. \& Sun, J. in 2015 IEEE International Conference on Computer Vision 1026-1034 (2015).

39. Krizhevsky, A., Sutskever, I. \& Hinton, G.E. in Advances in Neural Information Processing Systems 25: 26th Annual Conference on Neural Information Processing Systems 2012 1106-1114 (2012).

40. Shelhamer, E., Long, J. \& Darrell, T. Fully Convolutional Networks for Semantic Segmentation. IEEE Trans Pattern Anal Mach Inte// 39, 640-651 (2017).

41. Wang, Z., Simoncelli, E. \& Bovik, A. in ASILOMAR CONFERENCE ON SIGNALS SYSTEMS AND COMPUTERS (2002).

42. He, K., Zhang, X., Ren, S. \& Sun, J. in 2016 IEEE/CVF Conference on Computer Vision and Pattern Recognition (CVPR) (2016).

43. Zhang, H. et al. ResNeSt: Split-Attention Networks. (2020).

44. Ronneberger, O., Fischer, P. \& Brox, T. U-Net: Convolutional Networks for Biomedical Image Segmentation. Springer, Cham (2015).

45. Zhou, Z., Siddiquee, M.M.R., Tajbakhsh, N. \& Liang, J. UNet++: Redesigning Skip Connections to Exploit Multiscale Features in Image Segmentation. IEEE transactions on medical imaging 39, 1856-1867 (2020).

46. Kan, S., Zhang, Y., Zhang, F. \& Cen, Y. A GAN-Based Input-Size Flexibility Model for Single Image Dehazing. (2021).

47. Sun, H., Zhao, Z. \& He, Z. in 2020 IEEE/CVF Conference on Computer Vision and Pattern Recognition (CVPR) (2020).

48. Mirza, M. \& Osindero, S. Conditional Generative Adversarial Nets. (2014).

49. Wang, Z., Bovik, A.C., Sheikh, H.R. \& Simoncelli, E.P. Image quality assessment: from error visibility to structural similarity. IEEE Trans Image Process 13, 600-612 (2004).

50. Descloux, A., Grußmayer, K.S. \& Radenovic, A. Parameter-free image resolution estimation based on decorrelation analysis. Nat Methods 16, 918-924 (2019).

51. Bolte, S. \& Cordelières, F.P. A guided tour into subcellular colocalization analysis in light microscopy. J Microsc 224, 213-232 (2006) 
52. Bates, M., Huang, B., Dempsey, G.T. \& Zhuang, X. Multicolor super-resolution imaging with photo-switchable fluorescent probes. Science 317, 1749-1753 (2007).

53. Nakamura, S. et al. Dissecting the nanoscale distributions and functions of microtubule-end-binding proteins EB1 and ch-TOG in interphase HeLa cells. PLoS One 7, e51442 (2012).

54. Akhmanova, A. \& Steinmetz, M.O. Control of microtubule organization and dynamics: two ends in the limelight. Nat Rev Mol Cell Bio/ 16, 711-726 (2015).

55. Zhang, R., Alushin, G.M., Brown, A. \& Nogales, E. Mechanistic Origin of Microtubule Dynamic Instability and Its Modulation by EB Proteins. Ce// 162, 849-859 (2015).

56. Weigert, M. et al. Content-aware image restoration: pushing the limits of fluorescence microscopy. Nat Methods 15, 1090-1097 (2018).

57. Glorot, X., Bordes, A. \& Bengio, Y. in Journal of Machine Learning Research 315-323 (2011).

58. He, K., Zhang, X., Ren, S. \& Sun, J. Spatial Pyramid Pooling in Deep Convolutional Networks for Visual Recognition. IEEE Trans Pattern Anal Mach Inte// 37, 1904-1916 (2015).

59. Kingma, D.P. \& Ba, J. in 3rd International Conference on Learning Representations (ICLR 2015) (2015).

60. Zhou, W., Bovik, A.C., Sheikh, H.R. \& Simoncelli, E.P. Image quality assessment: from error visibility to structural similarity. IEEE Trans Image Process 13 (2004).

\section{Acknowledgments:}

We would like to thank Lamei Li and Baihai Huang for technical assistance, Ning Li and Dr Tao Wei for advice on super resolution microscopy. This work was supported by CAMS Innovation Fund for Medical Sciences (CIFMS) (2016-I2M-1-001) and National Science Foundation of China (81972572) to WJ, Tianjin Science and Technology Support Program Project (18YFZCSY00100) and New Century Excellent Talents in University in China (NCET-11-1066) to CZ and HAMD Funds for Health Research (2019-046-FW) to XY.

\section{Author contributions:}

LX and WJ designed research; LX, SK and YC designed the deep neural network; LX and SK performed research; LX, SK, XY, YP, YF, YL, CZ and WJ analyzed data and LX, SK and WJ wrote the paper.

\section{Declaration of interests:}

WJ and YL are the shareholders of HAMD (Ningbo) Intelligent Medical Technology Co. Ltd.

\section{Data and materials availability:}

The data presented here are tabulated in the main paper and supplementary materials. All data and code will be available based on the publication. Deep learning models reported in this work used standard libraries and scripts that publicly available in TensorFlow. 
Figure 1. The schematic architecture of X-Microscopy and the training strategy of URNet-8 and X-Net.

(A) The architecture of X-Microscopy. X-Microscopy was constructed with fusion of X-Net and UR-Net-8 that enabled fast and high-fidelity SRMs reconstruction from WFs alone.

(B) The neural network architecture and training strategy of UR-Net-8. UR-Net-8 was constructed to have a generator network $\mathcal{G}_{\mathrm{r}}$ and a discriminator network $\mathcal{D}_{\mathrm{r}}$ for performing adversarial training. Residual unit was utilized between each encoding unit and decoding unit. The WFs acquired from conventional microscopy were used as the input images, whereas the sparse STORM images $(k=10000)$ were served as the G_T. The training loss function was displayed as the combination of MS-SSIM loss, $l_{1}$ norm loss and generative adversarial loss.

(C) The architecture and training workflow of X-Net. The generator network $\mathcal{G}_{\mathrm{r}}$ displayed two parallel paths with an $X$-shape to share information reciprocally between encoders and decoders. The two desired inputs of $\mathcal{G}_{\text {r }}$, were set for generated sparse predicted from URNet-8 and/or WFs obtained from conventional microscopy. The attention and U-connection residual modules were established between each pair of encoding unit ei and decoding unit $\mathrm{d}(9-i)(1 \leqslant i \leqslant 8)$ based on skip connection, respectively. During training, the output of the generator $(\mathrm{XKj})$ was compared to the target image G_T (perfect) via three losses. The 'super-resolution reconstruction error' was determined by the difference between the XK3 and the target G_T using a combination of the L1, L2 and L3.

\section{Figure 2. Validating the feasibility and effectiveness of X-Net.}

(A) SRM reconstruction from undersampled STORM images (sparse) and/or WFs by X-Net and ANNA-PALM. The sparse images and/or WFs were used as the input images and the STORM images (perfect) served as the G_T. Representative WF, sparse ( $k=300)$, perfect and XK3 images of microtubules were shown. Image quality assessments (IQA) of the trained X-Net in terms of SSIM and resolution were displayed. Scale bar, $5 \mu \mathrm{m}$.

(B) Intensities along the lines as in (A) indicated by the yellow arrowheads in the images of WF, perfect and XK3 were shown.

(C) The heatmaps of cyan boxes as in (A) compared the true pixel intensity of perfect images to the network's predictions (XK3 images), with inset Pearson $r$ values. The bin width is 0.1 on a scale of zero to one (Methods). The numbers in the bins are frequency counts per 1,000 .

(D) SRM reconstruction with input-size flexibility by X-Net. Representative images of WF, sparse, perfect and XK3 images with various sizes $(75 \times 67,346 \times 304)$. Reconstruction quality of XK3 images was envaluated using SSIM.

(E) Statistical analysis and reconstruction quality assessment of XK3 images and output images of ANNA-PALM using SSIM. Image numbers in each group, $n=144$.

(F) Statistical analysis and reconstruction quality assessment of XK3 images using resolution. 
Image numbers in each group, $\mathrm{n}=144$.

(G) SRM reconstruction quality of STORM, X-Net and ANNA-PALM as function of frame number (or acquisition time) determined by SSIM were shown. Red curve: reconstruction quality of the standard STORM. Blue curve: reconstruction quality of X-Net using the sparse and WF as input. Image numbers in each group, $n=35$. Khaki curve: reconstruction quality of ANNA-PALM using the sparse and WF as input. Image numbers in each group, $n=35$.

\section{Figure 3. SRM determination by X-Microscopy using MTs and F-actin.}

(A) SRM reconstructions of microtubule (MT) images in U373MG cells using only WFs as input by X-Microscopy. WFs obtained from conventional microscopy were used as inputs to generate sparse by UR-Net-8. Subsequently, WFs and generated sparse severed as inputs via X-Net to output XK3 images. High-resolution details of SRM reconstructions were highlighted by arrows (red, green, pink and blue). Scale bar, $5 \mu \mathrm{m}$.

(B) Quantification of the similarity among WFs, perfect and XK3 images using intensity profile analysis. Intensity along the white line arrows were determined by Image $\mathrm{J}$ to demonstrate the reconstruction quality.

(C) Reconstructions of F-actin SRMs by X-Microscopy in RPE1 cells stained with phalloidin647. Representative images of WFs, perfect and XK3 were shown. The reconstruction details were indicated by arrows (yellow and blue) and white boxes. SSIM values were determined and displayed at the lower left part in XK3 images. Scale bar, $5 \mu \mathrm{m}$.

(D) Reconstructions of Keratin-14 (K-14) SRMs by X-Microscopy in D3 cells. Representative images of WFs, perfect and XK3 were shown. SSIM values was determined and displayed at the lower right part in XK3 images. Scale bar, $5 \mu \mathrm{m}$.

(E) Representative images of WF, generated sparse, XK3 in U2-OS cells stained with rabbit anti H2B. SSIM values were shown at the upper left part in XK3 images. Scale bar, $5 \mu \mathrm{m}$.

(F) Representative SRM images reconstructed from WF images of H3K9me3 in U2-OS cells using X-Microscopy were displayed. SSIM value was shown at the upper left part in XK3 images. Scale bar, $5 \mu \mathrm{m}$.

Figure 4. SRM reconstructions of various subcellular structures, i.e., EB1, Lamin B1 and kinetochores, by X-Microscopy

(A) EB1 SRM reconstructions from WFs in RPE1 cells using X-Microscopy. Representative images of WF, perfect and XK3 immunostained with anti-EB1 antibody were shown. Arrows (yellow, green, pink and blue) showed the reconstruction details. The reconstruction qualities were determined by SSIM metric and the calculated values were marked at the lower left of XK3 images. Experiments and analysis were repeated with 10 different images, showing similar results.

(B) The heatmap of white boxes as in (A) compared the true fluorescence pixel intensity to the network's predictions with an inset Pearson r value. Scale bar, $5 \mu \mathrm{m}$.

(C) Lamin B1 SRM reconstructions from WFs in U2-OS cells by X-Microscopy. The calculated SSIM value between G_T images and XK3 images were displayed in the XK3 images. 
Experiments and analysis were repeated with 20 different images, showing similar results. Scale bar, $5 \mu \mathrm{m}$.

(D) Representative images of WF, perfect and XK3 in U2-OS cells immunostained with antiCREST antibody were shown. Reconstruction details in XK3 images was referred by Arrows: blue, cyan, green and pink. The reconstruction qualities were calculated by SSIM and illustrated in XK3 images. Experiments and analysis were repeated with 10 different images, showing similar results. Scale bar, $5 \mu \mathrm{m}$.

(E) Reconstructions of microtubule SRMs by X-Microscopy under the perturbation conditions of STORM. Representative WF, perfect and XK3 images were shown. The perfect images were acquired using standard STORM with quenchable imaging buffer due to long acquisition time. Scale bar, $3 \mu \mathrm{m}$. The arrows in WF, XK3 and perfect images pointed to the reconstruction details in complex areas. Quantitative analysis of reconstruction quality was determined by error maps, where the brighter the value, the greater the gap between the reconstructed SRMs and the perfect images.

Figure 5. Reconstructions of EB1 and microtubule colocalization SRMs by X-Microscopy.

(A) Representative images of WF, generated sparse, XK3 in U2OS cells stained with anti- $\alpha-$ tubulin and anti EB1 antibodies and then stained with Alexa Fluor 546- and Atto 488conjugated secondary antibodies. WFs were captured with conventional microscopy. The merged images were shown in the left panel, where red indicated microtubule, green indicated EB1. Scale bars, $20 \mu \mathrm{m}$. XK3 images predicted by X-Microscopy with pre-trained microtubule and EB1 models were shown. White boxes in merged XK3 images represented the distribution of EB1 and microtubule signals.

(B) Line profiles of fluorescence intensity plotted from the white boxes along microtubules and EB1 were indicated.

\section{Figure 6. Multicolor SRM reconstructions by X-Microscopy.}

(A) Representative images of a WF and its corresponding SRM reconstructed by X-Microscopy were shown. The WF image was co-stained with anti-a-tubulin and Alexa Fluor 647phalloidin. Color map: red for F-actins, green for MTs. The WF images were acquired with conventional microscopy (100×/1.49-NA objective lens). The XK3 images were predicted by X-Microscopy with two pre-trained models of microtubule and F-actin. Scale bar, $20 \mu \mathrm{m}$. Resolution of the WF and the SRM determined by decorrelation analysis were indicated in the images.

(B) Representative images of WF in U2OS cells co-stained with anti-a-tubulin, anti-Lamin B1 and anti-CREST antibodies and XK3 images generated by X-Microscopy were displayed. Red: Lamin B1, Green: MTs, Grey: Kinetochore. Scale bar, $20 \mu \mathrm{m}$. Resolution of the SRM and WF determined by decorrelation analysis were indicated. Crisscross and bifurcation of complex regions in WF images along the white and pink arrowheads were clearly shown in XK3 images. 

objective lens.

(A) Representative images of WF and XK3 in U2-OS cells stained with Lamin B1 were shown. The WFs were acquired by Leica DM500B equipped with a 20×/0.50-NA objective, a $40 \times / 0.75-N A$ objective and a 100×/1.30-NA objective. The corresponding XK3 images were reconstructed by X-Microscopy with pre-trained model of Lamin B1. The resize algorithm was utilized to resize images to the same conversion value of training dataset $(0.05 \mu \mathrm{m} / \mathrm{px})$ before testing (for details, see materials and methods section).

(B) The input images were recorded by a Nikon eclipseTi2 microscope with $20 \times / 0.75-\mathrm{NA}$, a $40 \times / 0.95-N A, 60 \times / 1.40-N A, 100 \times / 1.49-N A$ objectives. The pretrained Lamin B1 model based on $100 \times / 1.49-N A$ (Nikon) were applied to reconstruct these input images, resulting in outputting the resolution enhanced SRMs. Note that the representative reconstructions of SRMs by X-Microscopy were shown at the lower right white boxes of the raw images.

(C) Representative WFs of Microtubule obtained by Nikon Ti2 eclipse equipped with 20×/0.75NA, 60×/1.40-NA and 100×/1.49-NA objectives and XK3 images were shown. Experiments and analysis were repeated with 10 different images.

(D) Representative WF of Microtubule obtained by Leica DM500B equipped with 100×/1.30NA objective and XK3 image were shown. 


\section{Figures}

A

widefield(WF)

X-Microscopy
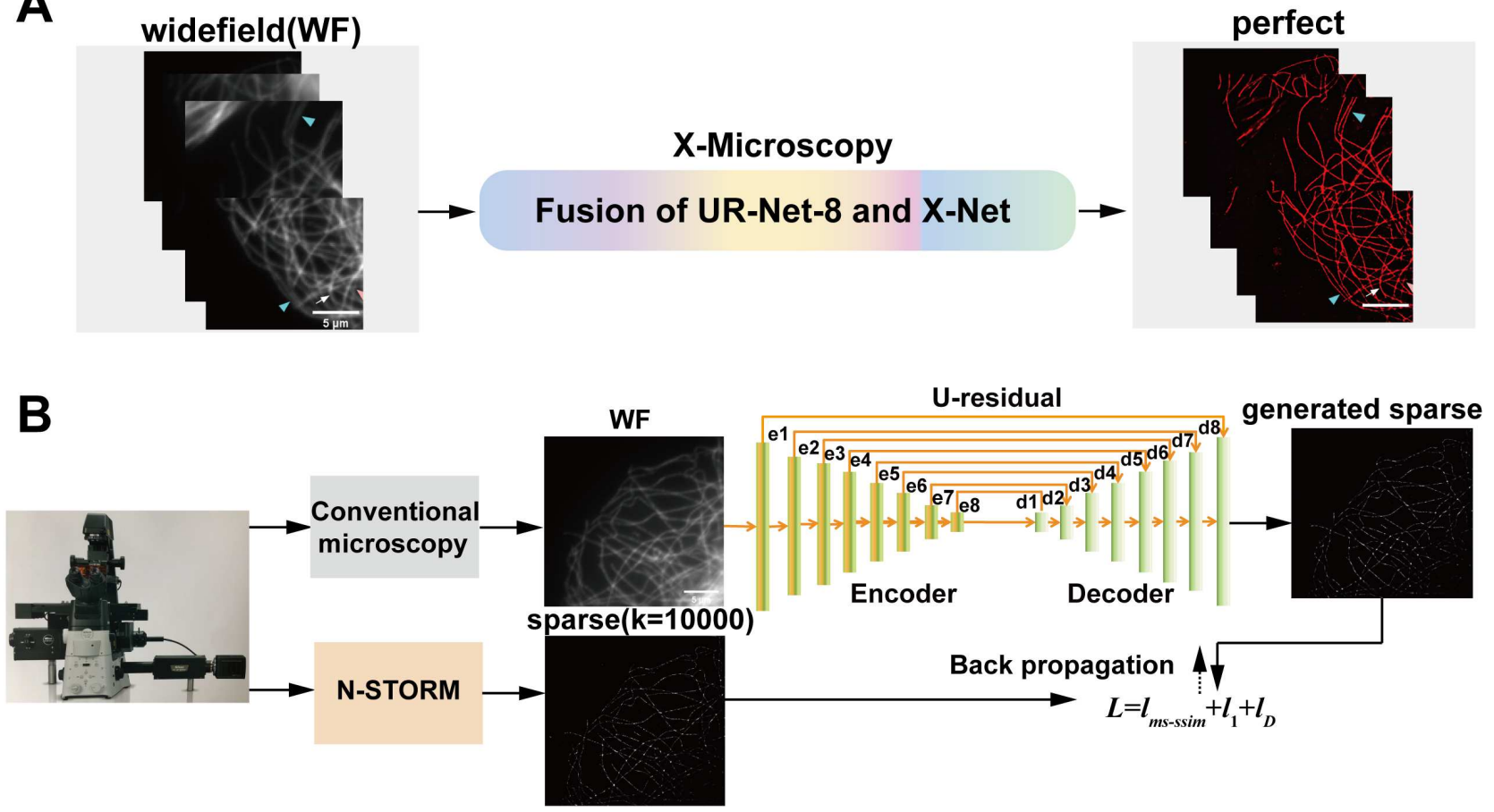

C
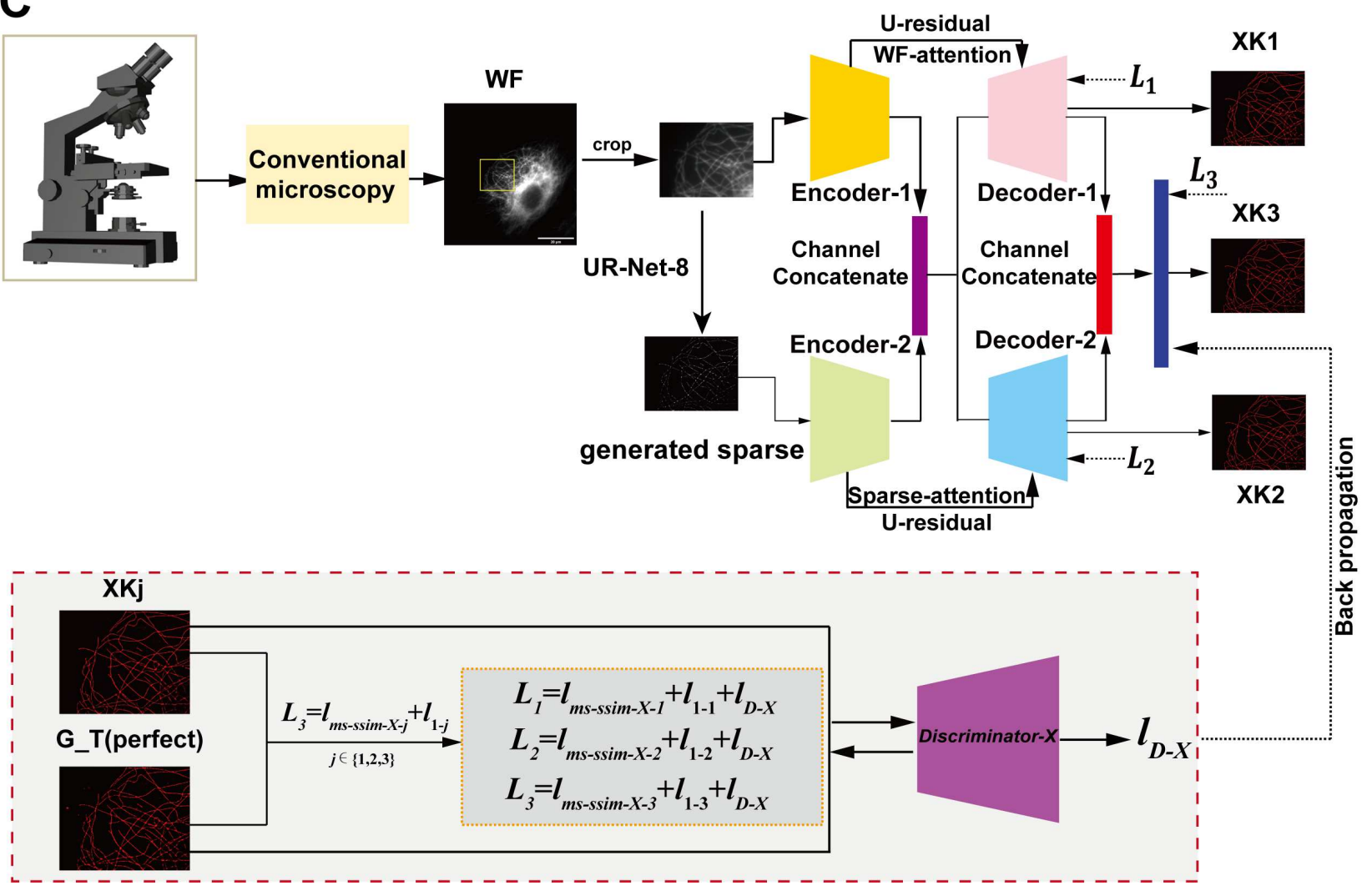

Figure 1

The schematic architecture of X-Microscopy and the training strategy of UR-Net-8 and X-Net. 
(A) The architecture of X-Microscopy. X-Microscopy was constructed with fusion of X-Net and UR-Net-8 that enabled fast and high-fidelity SRMs reconstruction from WFs alone.

(B) The neural network architecture and training strategy of UR-Net-8. UR-Net-8 was constructed to have a generator network $\mathbb{V}_{\mathrm{r}}$ and a discriminator network $\mathbb{\nabla}_{\mathrm{r}}$ for performing adversarial training. Residual unit was utilized between each encoding unit and decoding unit. The WFs acquired from conventional microscopy were used as the input images, whereas the sparse STORM images $(k=10000)$ were served as the G_T. The training loss function was displayed as the combination of MS-SSIM loss, F norm loss and generative adversarial loss.

(C) The architecture and training workflow of X-Net. The generator network $\nabla_{\mathrm{r}}$ displayed two parallel paths with an X-shape to share information reciprocally between encoders and decoders. The two desired inputs of $\mathbb{\nabla}_{\mathrm{r}}$, were set for generated sparse predicted from UR-Net-8 and/or WFs obtained from conventional microscopy. The attention and U-connection residual modules were established between each pair of encoding unit ei and decoding unit $\mathrm{d}(9-i)(1 \leq i \leq 8)$ based on skip connection, respectively. During training, the output of the generator $(\mathrm{XK} J)$ was compared to the target image G_T (perfect) via three losses. The 'super-resolution reconstruction error' was determined by the difference between the XK3 and the target G_T using a combination of the L1, L2 and L3. 
A

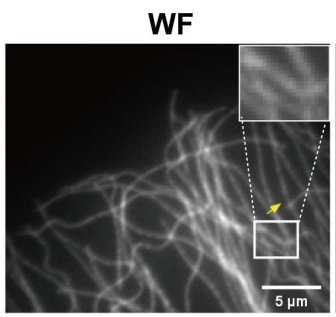

$\operatorname{sparse}(K=300)$

perfect

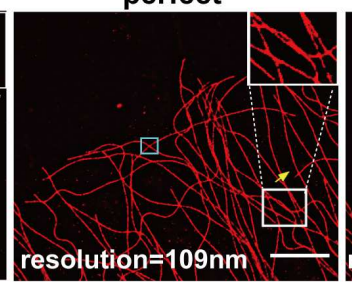

XK3(input=sparse)

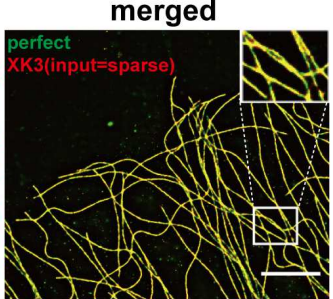

resolution $=106 \mathrm{~nm}$

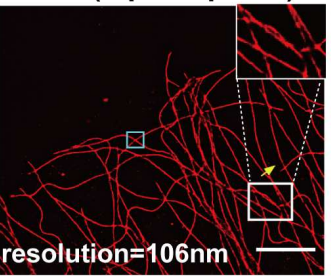

XK3(input=WF+sparse)

merged

ANNA-PALM(input=sparse) input=WF+sparse

\begin{tabular}{|c|c|c|}
\hline \multicolumn{3}{|c|}{ IQA } \\
\hline SSIM & X-Net & $\begin{array}{l}\text { ANNA- } \\
\text { PALM }\end{array}$ \\
\hline $\begin{array}{l}\text { input= } \\
\text { sparse }\end{array}$ & 0.928 & 0.802 \\
\hline $\begin{array}{c}\text { input= } \\
\text { sparse }+W F\end{array}$ & 0.954 & 0.809 \\
\hline
\end{tabular}

resolution $=105 \mathrm{~nm}$
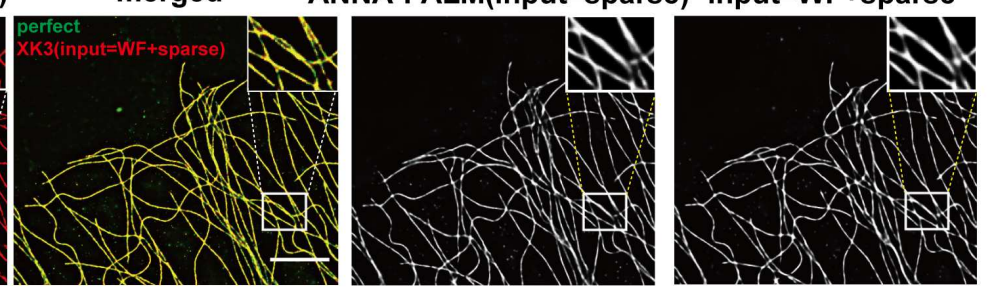

B

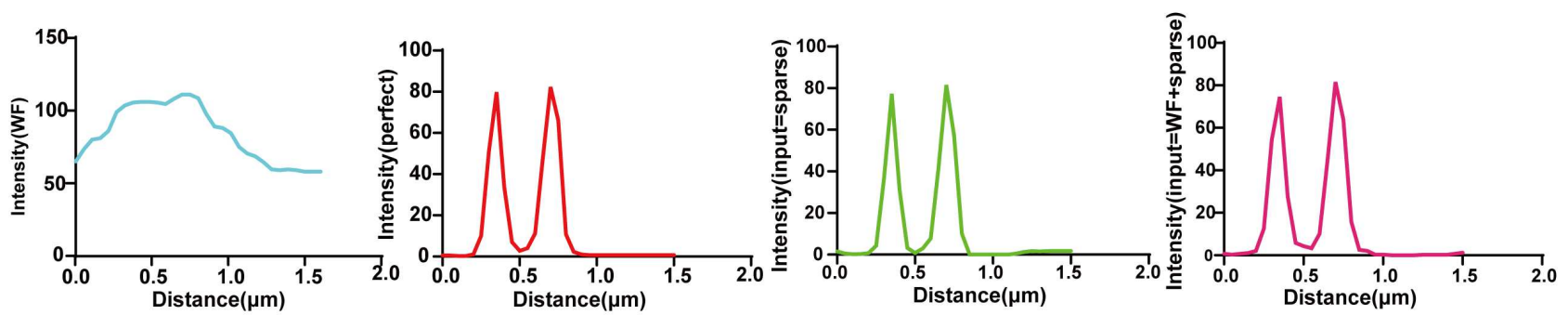

C
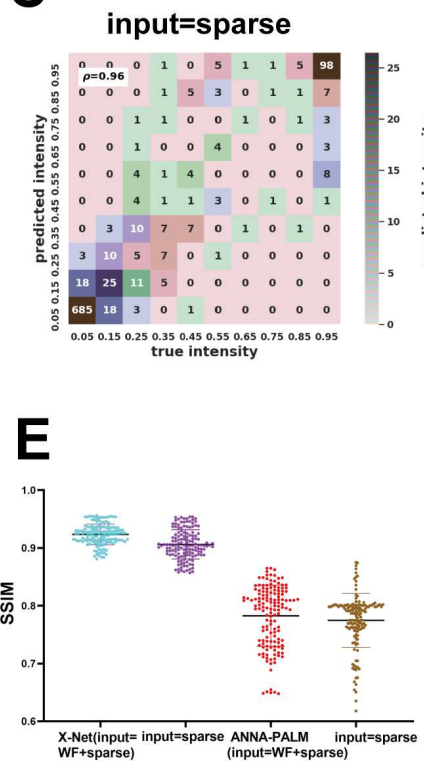

input $=W F+s p a r s e$

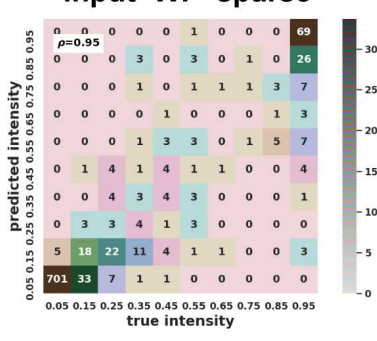

D
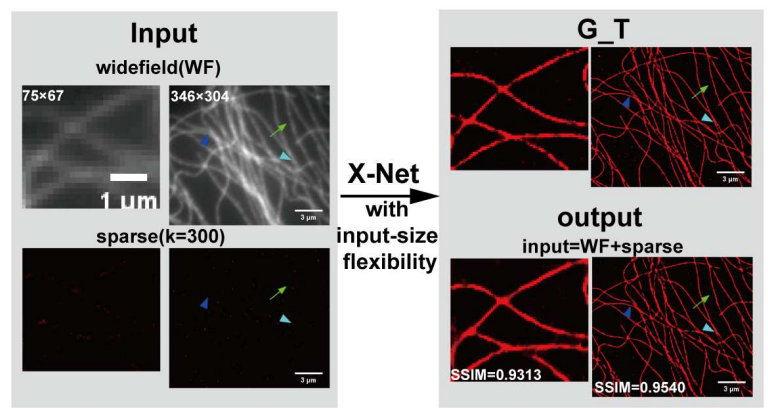

$\mathbf{F}$

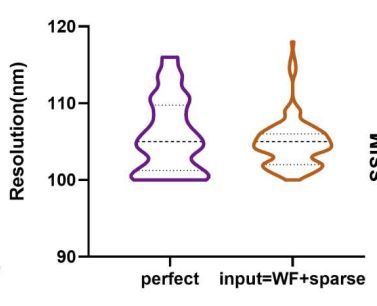

G

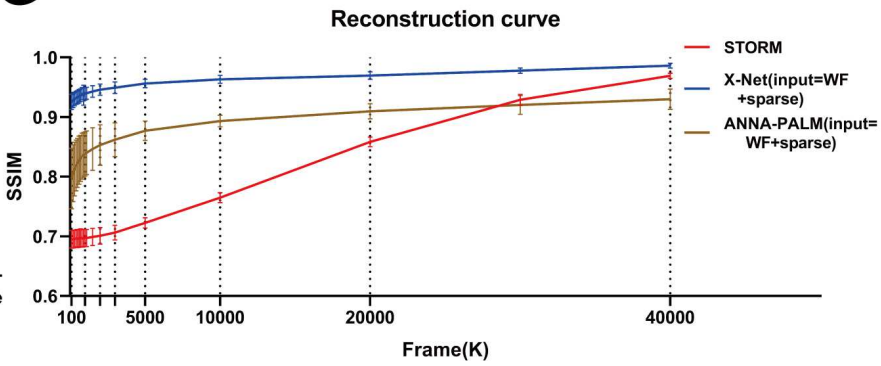

Figure 2

Validating the feasibility and effectiveness of X-Net.

(A) SRM reconstruction from undersampled STORM images (sparse) and/or WFs by X-Net and ANNAPALM. The sparse images and/or WFs were used as the input images and the STORM images (perfect) served as the G_T. Representative WF, sparse $(k=300)$, perfect and XK3 images of microtubules were 
shown. Image quality assessments (IQA) of the trained X-Net in terms of SSIM and resolution were displayed. Scale bar, $5 \mu \mathrm{m}$.

(B) Intensities along the lines as in (A) indicated by the yellow arrowheads in the images of WF, perfect and XK3 were shown.

(C) The heatmaps of cyan boxes as in (A) compared the true pixel intensity of perfect images to the network's predictions (XK3 images), with inset Pearson $r$ values. The bin width is 0.1 on a scale of zero to one (Methods). The numbers in the bins are frequency counts per 1,000.

(D) SRM reconstruction with input-size flexibility by X-Net. Representative images of WF, sparse, perfect and XK3 images with various sizes $(75 \times 67,346 \times 304)$. Reconstruction quality of XK3 images was envaluated using SSIM.

(E) Statistical analysis and reconstruction quality assessment of XK3 images and output images of ANNA-PALM using SSIM. Image numbers in each group, $n=144$.

(F) Statistical analysis and reconstruction quality assessment of XK3 images using resolution. Image numbers in each group, $\mathrm{n}=144$.

(G) SRM reconstruction quality of STORM, X-Net and ANNA-PALM as function of frame number (or acquisition time) determined by SSIM were shown. Red curve: reconstruction quality of the standard STORM. Blue curve: reconstruction quality of X-Net using the sparse and WF as input. Image numbers in each group, $n=35$. Khaki curve: reconstruction quality of ANNA-PALM using the sparse and WF as input. Image numbers in each group, $n=35$.

\section{Figure 3}

\section{SRM determination by X-Microscopy using MTs and F-actin.}

(A) SRM reconstructions of microtubule (MT) images in U373MG cells using only WFs as input by XMicroscopy. WFs obtained from conventional microscopy were used as inputs to generate sparse by URNet-8. Subsequently, WFs and generated sparse severed as inputs via X-Net to output XK3 images. Highresolution details of SRM reconstructions were highlighted by arrows (red, green, pink and blue). Scale bar, $5 \mu \mathrm{m}$.

(B) Quantification of the similarity among WFs, perfect and XK3 images using intensity profile analysis. Intensity along the white line arrows were determined by Image $\mathrm{J}$ to demonstrate the reconstruction quality.

(C) Reconstructions of F-actin SRMs by X-Microscopy in RPE1 cells stained with phalloidin-647.

Representative images of WFs, perfect and XK3 were shown. The reconstruction details were indicated by 
arrows (yellow and blue) and white boxes. SSIM values were determined and displayed at the lower left part in XK3 images. Scale bar, $5 \mu \mathrm{m}$.

(D) Reconstructions of Keratin-14 (K-14) SRMs by X-Microscopy in D3 cells. Representative images of WFs, perfect and XK3 were shown. SSIM values was determined and displayed at the lower right part in XK3 images. Scale bar, $5 \mu \mathrm{m}$.

(E) Representative images of WF, generated sparse, XK3 in U2-OS cells stained with rabbit anti H2B. SSIM values were shown at the upper left part in XK3 images. Scale bar, $5 \mu \mathrm{m}$.

(F) Representative SRM images reconstructed from WF images of H3K9me3 in U2-OS cells using XMicroscopy were displayed. SSIM value was shown at the upper left part in XK3 images. Scale bar, $5 \mu \mathrm{m}$. 
A

perfect

XK3(input=WF)

XK3(input=generated sparse) $\mathrm{XK} 3$ (input=WF+generated sparse)
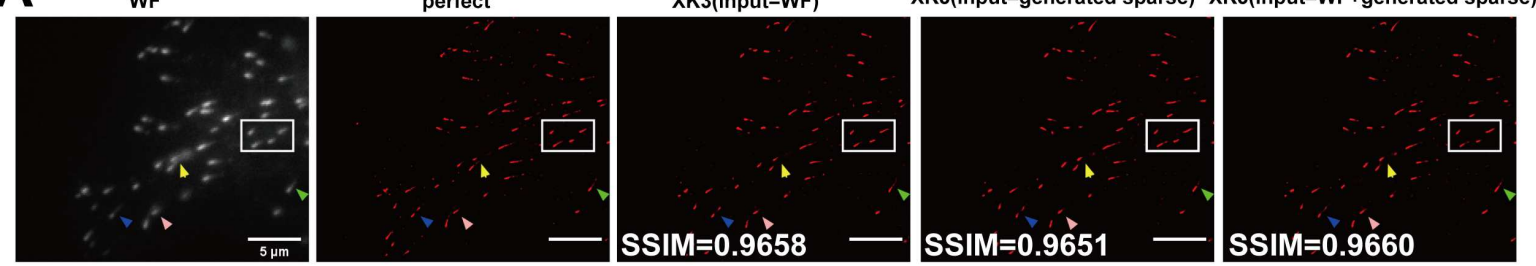

B
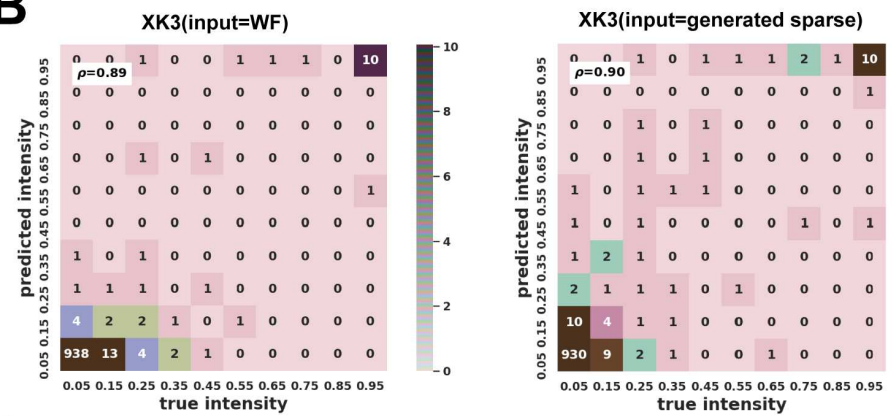

XK3(input=WF+generated sparse)
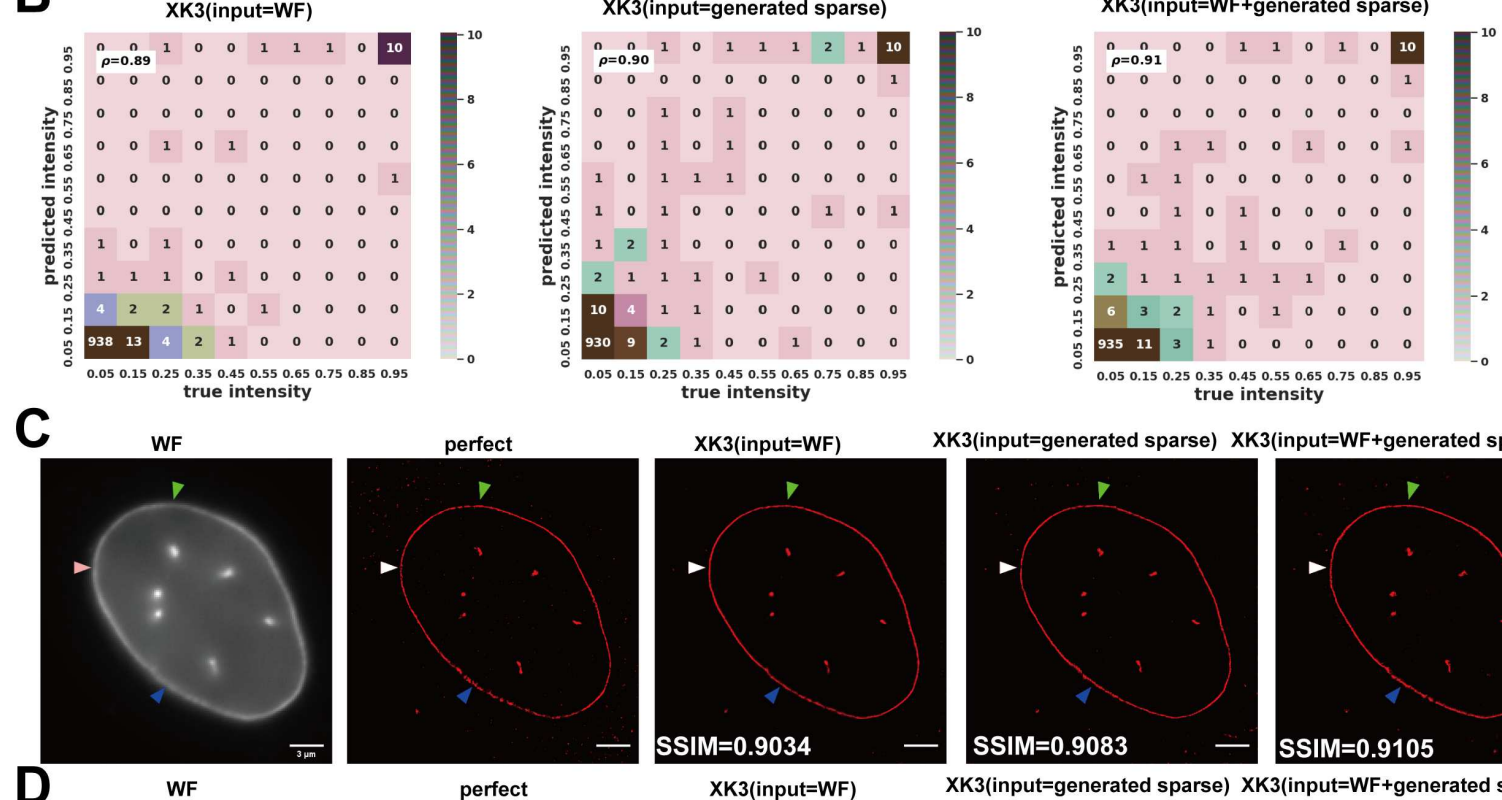

XK3(input=generated sparse) $\quad$ XK3(input=WF+generated sparse)
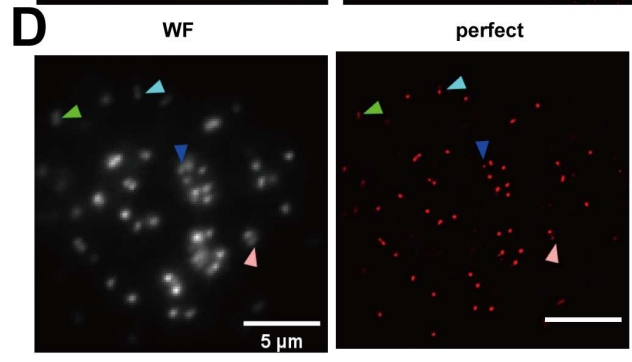

XK3(input=WF)
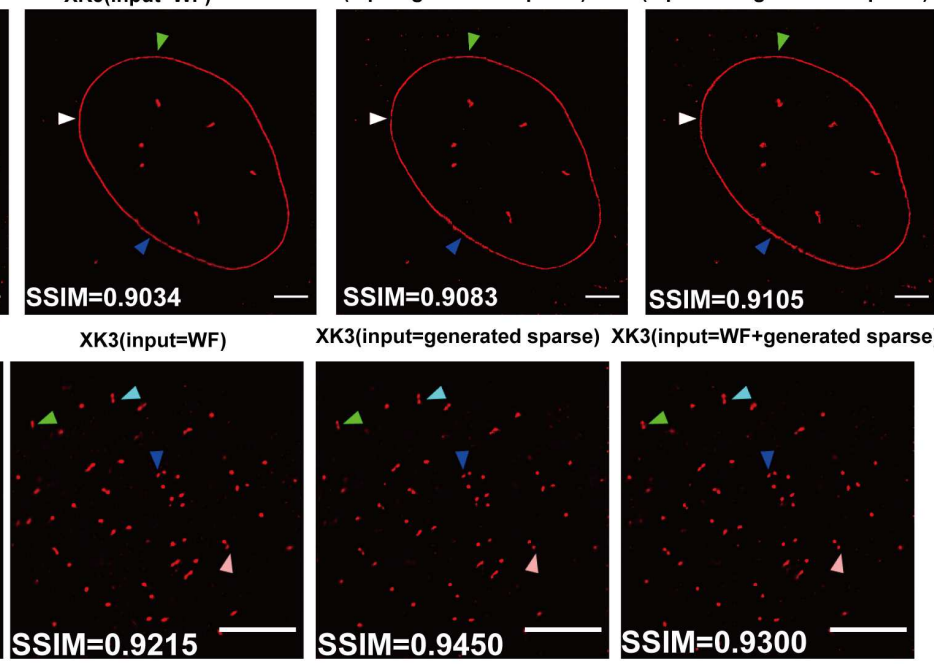

XK3(input=generated sparse) XK3(input=WF+generated sparse)

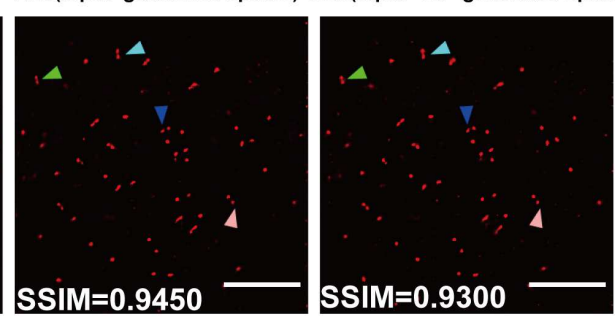

E
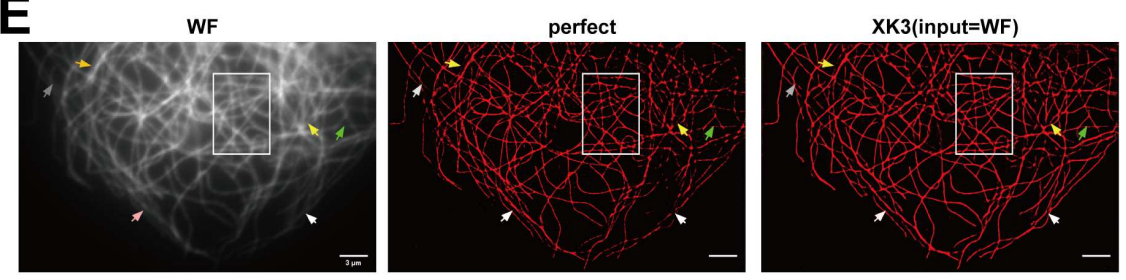

XK3(input=generated sparse)

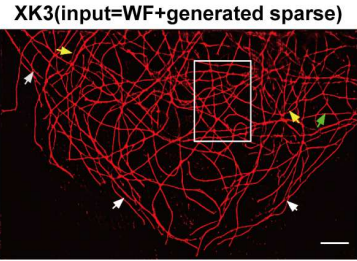

error map (input=WF)

error map(input=generated sparse) error map(input=WF+generated sparse)
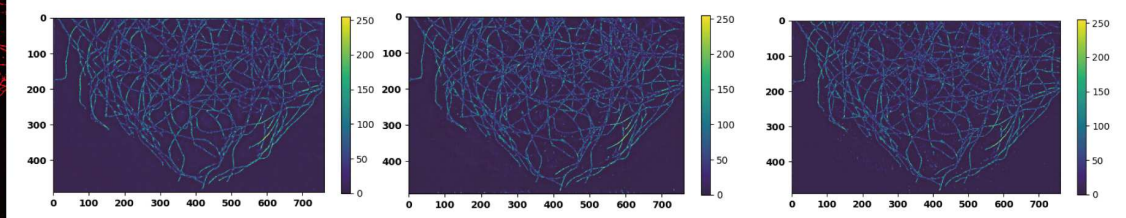

Figure 4

SRM reconstructions of various subcellular structures, i.e., EB1, Lamin B1 and kinetochores, by XMicroscopy

(A) EB1 SRM reconstructions from WFs in RPE1 cells using X-Microscopy. Representative images of WF, perfect and XK3 immunostained with anti-EB1 antibody were shown. Arrows (yellow, green, pink and 
blue) showed the reconstruction details. The reconstruction qualities were determined by SSIM metric and the calculated values were marked at the lower left of XK3 images. Experiments and analysis were repeated with 10 different images, showing similar results.

(B) The heatmap of white boxes as in (A) compared the true fluorescence pixel intensity to the network's predictions with an inset Pearson $r$ value. Scale bar, $5 \mu \mathrm{m}$.

(C) Lamin B1 SRM reconstructions from WFs in U2-OS cells by X-Microscopy. The calculated SSIM value between G_T images and XK3 images were displayed in the XK3 images. Experiments and analysis were repeated with 20 different images, showing similar results. Scale bar, $5 \mu \mathrm{m}$.

(D) Representative images of WF, perfect and XK3 in U2-OS cells immunostained with anti-CREST antibody were shown. Reconstruction details in XK3 images was referred by Arrows: blue, cyan, green and pink. The reconstruction qualities were calculated by SSIM and illustrated in XK3 images. Experiments and analysis were repeated with 10 different images, showing similar results. Scale bar, $5 \mu \mathrm{m}$.

(E) Reconstructions of microtubule SRMs by X-Microscopy under the perturbation conditions of STORM. Representative WF, perfect and XK3 images were shown. The perfect images were acquired using standard STORM with quenchable imaging buffer due to long acquisition time. Scale bar, $3 \mu \mathrm{m}$. The arrows in WF, XK3 and perfect images pointed to the reconstruction details in complex areas. Quantitative analysis of reconstruction quality was determined by error maps, where the brighter the value, the greater the gap between the reconstructed SRMs and the perfect images. 


\section{A}

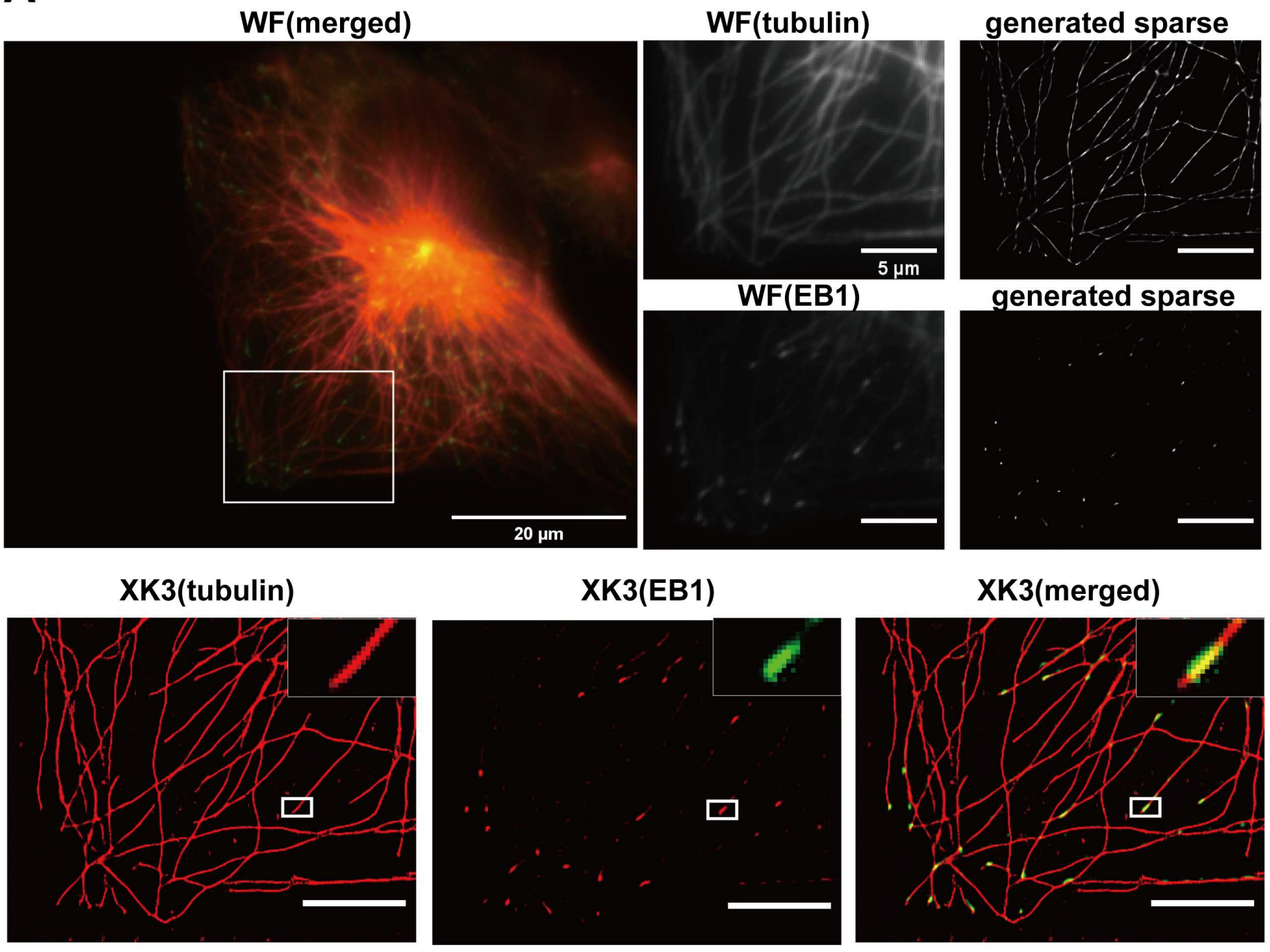

B

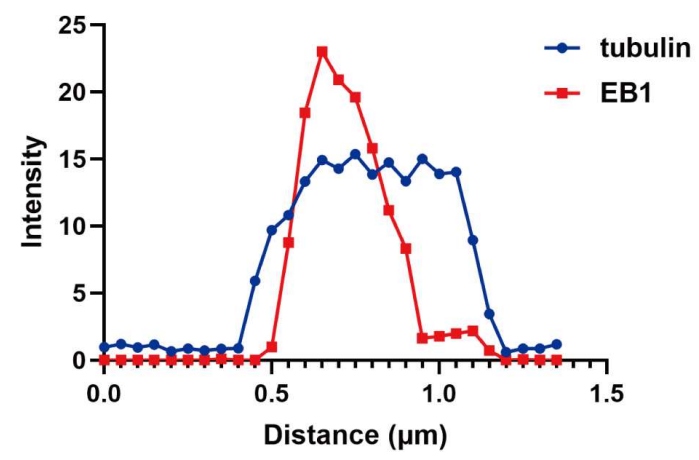

Figure 5

Reconstructions of EB1 and microtubule colocalization SRMs by X-Microscopy.

(A) Representative images of WF, generated sparse, XK3 in U2OS cells stained with anti-a-tubulin and anti EB1 antibodies and then stained with Alexa Fluor 546- and Atto 488-conjugated secondary antibodies. WFs were captured with conventional microscopy. The merged images were shown in the left 
panel, where red indicated microtubule, green indicated EB1. Scale bars, $20 \mu \mathrm{m}$. XK3 images predicted by X-Microscopy with pre-trained microtubule and EB1 models were shown. White boxes in merged XK3 images represented the distribution of EB1 and microtubule signals.

(B) Line profiles of fluorescence intensity plotted from the white boxes along microtubules and EB1 were indicated.

\section{Figure 6}

\section{Multicolor SRM reconstructions by X-Microscopy.}

(A) Representative images of a WF and its corresponding SRM reconstructed by X-Microscopy were shown. The WF image was co-stained with anti-a-tubulin and Alexa Fluor 647-phalloidin. Color map: red for F-actins, green for MTs. The WF images were acquired with conventional microscopy (100x/1.49-NA objective lens). The XK3 images were predicted by X-Microscopy with two pre-trained models of microtubule and F-actin. Scale bar, $20 \mu \mathrm{m}$. Resolution of the WF and the SRM determined by decorrelation analysis were indicated in the images.

(B) Representative images of WF in U2OS cells co-stained with anti-a-tubulin, anti-Lamin B1 and antiCREST antibodies and XK3 images generated by X-Microscopy were displayed. Red: Lamin B1, Green: MTs, Grey: Kinetochore. Scale bar, $20 \mu \mathrm{m}$. Resolution of the SRM and WF determined by decorrelation analysis were indicated. Crisscross and bifurcation of complex regions in WF images along the white and pink arrowheads were clearly shown in XK3 images.

\section{Figure 7}

\section{SRM reconstructions by X-Microscopy using different microscopic systems and objective lens.}

(A) Representative images of WF and XK3 in U2-OS cells stained with Lamin B1 were shown. The WFs were acquired by Leica DM500B equipped with a 20×/0.50-NA objective, a 40×/0.75-NA objective and a $100 \times / 1.30-N A$ objective. The corresponding XK3 images were reconstructed by X-Microscopy with pretrained model of Lamin B1. The resize algorithm was utilized to resize images to the same conversion value of training dataset $(0.05 \mu \mathrm{m} / \mathrm{px})$ before testing (for details, see materials and methods section).

(B) The input images were recorded by a Nikon eclipseTi2 microscope with 20×/0.75-NA, a 40×/0.95-NA, 60x/1.40-NA, 100×/1.49-NA objectives. The pretrained Lamin B1 model based on 100x/1.49-NA (Nikon) were applied to reconstruct these input images, resulting in outputting the resolution enhanced SRMs. Note that the representative reconstructions of SRMs by X-Microscopy were shown at the lower right white boxes of the raw images. 
(C) Representative WFs of Microtubule obtained by Nikon Ti2 eclipse equipped with 20x/0.75-NA, $60 \times / 1.40-N A$ and 100x/1.49-NA objectives and XK3 images were shown. Experiments and analysis were repeated with 10 different images.

(D) Representative WF of Microtubule obtained by Leica DM500B equipped with 100x/1.30-NA objective and XK3 image were shown.

\section{Supplementary Files}

This is a list of supplementary files associated with this preprint. Click to download.

- 2021.01.13SupplementaryinformationNC.pdf 